\title{
Computational study of the rovibrational spectrum of $\mathrm{H}_{2} \mathrm{O}-\mathrm{HF}$
}

\author{
Dominika Viglaska* \\ Groupe de Spectrométrie Moléculaire et Atmosphérique UMR CNRS 7331, \\ UFR Sciences BP 1039,51687 Reims Cedex 2, France \\ Xiao-Gang Wang ${ }^{\dagger}$ and Tucker Carrington Jr. ${ }^{\ddagger}$ \\ Chemistry Department, Queen's University, \\ Kingston, Ontario KYL 3N6, Canada \\ David P. Tew ${ }^{\S}$ \\ Physical and Theoretical Chemistry Laboratory, \\ University of Oxford, South Parks Road, Oxford, OX1 3QZ, UK
}

\begin{abstract}
In this paper we report rovibrational energy levels, transition frequencies, and intensities computed for $\mathrm{H}_{2} \mathrm{O}-\mathrm{HF}$ using a new ab initio potential energy surface and compare with available experimental data. We use the rigid monomer approximation. A $G_{4}$ symmetry-adapted Lanczos algorithm and an uncoupled product basis are employed. The rovibrational levels are computed up to $J=4$. The new analytic 9-D potential is fit to 39771 counterpoise corrected $\operatorname{CCSD}(\mathrm{T})\left(\mathrm{F} 12^{*}\right) /$ augcc-pVTZ energies and reduces to the sum of uncoupled $\mathrm{H}_{2} \mathrm{O}$ and HF potentials in the dissociation limit. On the new potential better agreement with experiment is obtained by re-assigning the $\mathrm{R}(1)$ transitions of two vibrational states.
\end{abstract}

\footnotetext{
*Electronic address: dominika.viglaska@univ-reims.fr

$\dagger$ Electronic address: xgwang.dalian@gmail.com

${ }^{\ddagger}$ Electronic address: Tucker. Carrington@queensu.ca, Fax:613-533-6669

$\S$ Electronic address: david.tew@chem.ox.ac.uk
} 


\section{INTRODUCTION}

Molecular complexes play an important role in the chemistry of planetary atmospheres[1]. In this paper, we report a theoretical study of ro-vibrational energy levels of $\mathrm{H}_{2} \mathrm{O}-\mathrm{HF}$, which is of considerable interest. There are many previous publications [2-16]. Excited intermolecular low energy states have been observed and it is therefore possible to test extensive regions of the potential energy surface (PES). The spectrum is complicated by a low inversion barrier and tunnelling between two global minima. The barrier height is estimated from experimental data and a 1-D model to be about $70 \mathrm{~cm}^{-1}[7]$. The global minima are pyramidal and have $C_{s}$ symmetry. The saddle point is planar and has $C_{2 v}$ symmmetry.

From the intensities of hot band progressions in the band associated with the HF stretching vibration in the complex, Thomas et al.[3] obtained the fundamental transition frequencies associated with H-bond deformations: $145 \pm 50 \mathrm{~cm}^{-1}$ for the out of plane bending mode $\nu_{\beta(o)} ; 170 \pm 50 \mathrm{~cm}^{-1}$ for the in plane bending mode $\nu_{\beta(i)}$; and at $180 \pm 30 \mathrm{~cm}^{-1}$ for intermolecular stretching $\nu_{\sigma}$. These results have large uncertainties reflecting the poor quality of the experimental method. However, two higher $\left(\nu_{B(o)}=666 \pm 30 \mathrm{~cm}^{-1}\right.$ and $\nu_{B(i)}=696 \pm$ $30 \mathrm{~cm}^{-1}$ ) bending vibration frequencies were better determined by analyzing the partially rotational-resolved low-resolution infrared absorption spectra. The results for these two bands are still the most accurately determined experimental data and will be compared to our calculated results. Bevan, Kisiel, and Legon et al. [2, 6, 7, 17] used relative intensities of the microwave transitions to estimate the frequencies of three intermolecular modes: $\nu_{\beta(o)}=$ $64 \pm 10 \mathrm{~cm}^{-1} ; \nu_{\beta(i)}=157 \pm 10 \mathrm{~cm}^{-1} ;$ and $\nu_{\sigma}=176 \pm 15 \mathrm{~cm}^{-1}$. The uncertainties in the Kisiel et al. experimental values are smaller than those of Thomas et al.[3] but still large because the method they used to obtain the vibrational energies involves approximations. Future direct measurements of the far-infrared transitions to the vibrational states are necessary to obtain the accurate vibrational energies. However, the microwave $R(1)$ triplet lines of many vibrational states are assigned, but some vibrational state assignments are uncertain. These valuable experimental data provide an opportunity to test the accuracy of theoretical spectrum calculated in this work. In this paper, we use the notation of previous papers for the intermolecular modes[7]. In 2006, Belov et al. [16] published observed rotational frequencies up to $J=24$ for the ground state and one low-lying excited vibrational states obtained from 
a microwave study.

The purpose of this paper is to present a new 9-D $\mathrm{H}_{2} \mathrm{O}-\mathrm{HF}$ potential energy surface (PES) and calculated ro-vibrational frequencies, with corresponding symmetry labels, and to compare them with experimental counterparts. To compute the spectrum the monomer geometries are fixed at vibrationally averaged values and therefore we treat only five vibrations. The intermolecular frequencies are much lower than intramolecular frequencies which justifies this approximation. We use a Lanczos method and a large product basis.[18, 19]

In a very recent publication, Loreau et al. [20] presented the first global five-dimensional ab initio $\mathrm{H}_{2} \mathrm{O}-\mathrm{HF}$ potential energy surface. They calculated a few ro-vibrational states and compared with experiment. We compute more vibrational states and states with $J$ values as large as 4 . The $a b$ initio method they used is the same as ours. The PES of this paper is fit with an entirely different method. Our PES is 9-D, whereas the Loreau et al. is 5-D. We use the same coordinates and the same primitive basis functions as Loreau et al.. However our method differs from theirs in several respects: 1) they use Clebsch-Gordan coefficients to couple the basis functions and we do not; 2) we use the Lanczos method to solve the matrix eigenvalue problem without storing a Hamiltonian matrix; 3) we use quadrature for potential matrix elements and they expand the PES in their basis functions so that they can calculate matrix elements using exact formula. [21] We use wavefunction plots and symmetry arguments to assign vibrational levels to the states we compute. We also compute intensities which is useful for comparing with the microwave spectrum of Kisiel et al. [6-8].

\section{COMPUTATIONAL METHOD}

We use the kinetic energy operator (KEO) discussed initially by Brocks et al. [22]. It is written in terms of angular momentum operators defined in monomer- and dimer-fixed frames. The eight coordinates $\left(\alpha, \beta, \alpha_{A}, \beta_{A}, \gamma_{A}, \alpha_{B}, \beta_{B}\right.$ and $\left.r_{0}\right)$, where $\mathrm{A}=\mathrm{H}_{2} \mathrm{O}$ and $\mathrm{B}=\mathrm{HF}$, are illustrated in Figure 1. The $z$ axis of the dimer-fixed (DF) frame is along the intermonomer vector. The monomer-fixed (MF) frame for $\mathrm{HF}$ is obtained by rotating the DF frame first about $z$-axis by $\alpha_{B}$ (leading to the red dashed frame in Fig. 1), and then about the $y$-axis by $\beta_{B}$. The MF frame attached to $\mathrm{H}_{2} \mathrm{O}$ is obtained by rotating the $\mathrm{DF}$ frame by three successive angles $\left(\alpha_{A}, \beta_{A}, \gamma_{A}\right)$ following the $z-y$ - $z$ convention [23]. The monomer-fixed 

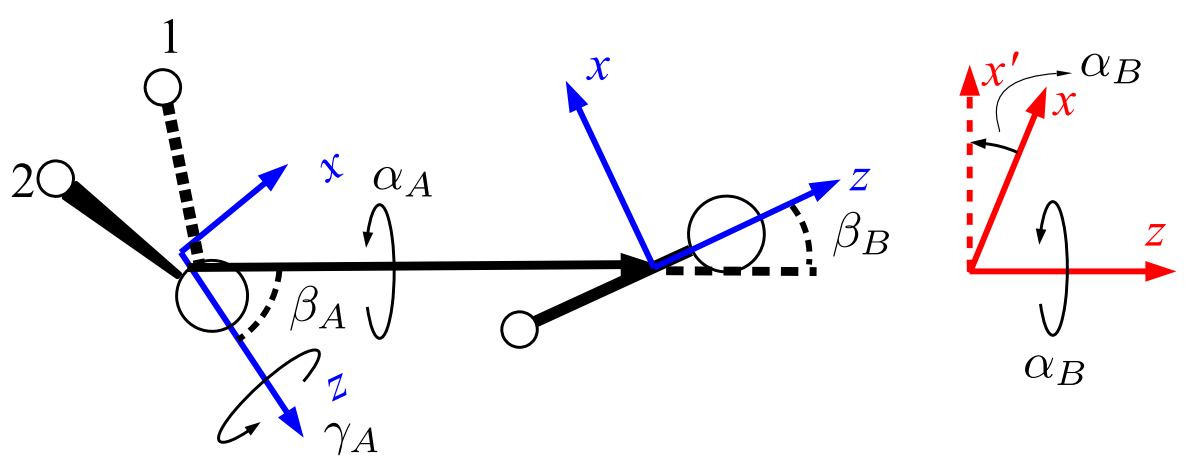

FIG. 1: The dimer-fixed coordinates used to compute rovibrationnal levels of $\mathrm{HF}-\mathrm{H}_{2} \mathrm{O}$. The monomer-fixed (MF) frames attached to $\mathrm{H}_{2} \mathrm{O}$ and $\mathrm{HF}$, respectively, are marked in blue. The DF frame is marked in red. The MF frame of HF is obtained by rotating the DF frame first about $z$-axis by $\alpha_{B}$ (leading to the red dashed frame) and then about the $y$-axis by $\beta_{B}$. The MF frame of $\mathrm{H}_{2} \mathrm{O}$ is obtained by rotating the DF frame first about $z$-axis by $\alpha_{A}$ and then about the $y$-axis by $\beta_{A}$, and finally about the $z$-axis by $\gamma_{A}$.

frame for $\mathrm{H}_{2} \mathrm{O}$ is a bisector frame with the $z$-axis along the bisector and the $x$-axis in the $\mathrm{H}_{2} \mathrm{O}$ molecular plane. The $z$ coordinate of $\mathrm{O}$ is positive and the $x$ coordinate of $\mathrm{H}_{1}$ is positive. The dashed $\mathrm{OH}$ bond is below the plane containing $\mathrm{HF}$ and $\mathrm{O}$.

The basis functions we use are products of parity adapted functions and discrete variable representation (DVR) function [24-26] for the intermonomer distance $r_{0}$. The parity adapted functions are linear combinations of uncoupled product functions, denoted $\left|j_{A} k_{A} m_{A} ; j_{B}\left(m_{B}\right) ; J K M\right\rangle[18,19,27-29]$, which are

$$
\sqrt{\left(\frac{2 j_{A}+1}{8 \pi^{2}}\right)\left(\frac{2 j_{B}+1}{4 \pi}\right)\left(\frac{2 J+1}{4 \pi}\right)} D_{m_{A} k_{A}}^{* j_{A}}\left(\alpha_{A}, \beta_{A}, \gamma_{A}\right) D_{m_{B} 0}^{* j_{B}}\left(\alpha_{B}, \beta_{B}, 0\right) D_{M K}^{* J}(\alpha, \beta, 0)
$$

and subject to the constraint $K \equiv m_{A}+m_{B} . D_{M K}^{* J}$ is a Wigner function.

Basis functions with even (odd) values of $k_{a}$ transform like the A (B) irreducible representation of the $G_{4}$ PI group. $G_{4}$ contains four operations, $G_{4}=\left\{E, E^{*}\right\} \times\{(12)\}$, where $\left\{E, E^{*}\right\}$ is the inversion group and (12) corresponds to the exchange of the two identical hydrogen atoms of the water monomer. 


\section{POTENTIAL ENERGY SURFACE}

\section{A. Electronic Structure}

Tables I and II list the geometric parameters and harmonic wavenumbers of the $C_{s}$ and $C_{2 v}$ stationary points optimised at the $\mathrm{fc}-\mathrm{CCSD}(\mathrm{T})\left(\mathrm{F} 12^{*}\right)$ level of theory[30-32] with the aug-cc-pVXZ basis sets[33, 34] X=D,T,Q and using the frozen core (fc) approximation. We use augmented basis sets because diffuse functions have been found to be important for an accurate description of systems containing a fluorine atom and are also recommended for use with F12 theory.[35, 36] The soft intermolecular potential is evidently sensitive to the basis set used for the correlation treatment even when using the F12 method.

Table III reports how the energy difference between the $C_{s}$ and $C_{2 v}$ stationary points on the $\operatorname{CCSD}(\mathrm{T})\left(\mathrm{F} 12^{*}\right)$ /aug-cc-pVTZ potential changes when evaluating the energy with larger basis sets, when applying a counterpoise correction and when adding corrections for core-valence correlation and higher-body correlation effects. With the F12 method, the basis set effect is saturated at quadruple-zeta level. Application of a counterpoise correction is important for an accurate description of the potential energy surface when using the lowercost aug-cc-pVTZ basis. The shift in relative energy of the two characteristic points on the potential surface due to post basis set limit fc-CCSD $(\mathrm{T})$ contributions is small. As is often observed[37] these effects often cancel each other and in this case the counterpoise corrected fc-CCSD $(\mathrm{T})\left(\mathrm{F} 12^{*}\right) /$ aug-cc-pVTZ energy difference is within $1 \mathrm{~cm}^{-1}$ of the most accurate value of $109 \mathrm{~cm}^{-1}$. To facilitate evaluation of the thousands of single point energies required as data points when fitting a global 9-D potential energy surface, we therefore use the counterpoise corrected frozen-core $\operatorname{CCSD}(\mathrm{T})\left(\mathrm{F} 12^{*}\right)$ method with an aug-cc-pVTZ basis.

\section{B. Data point generation}

A data set of a total of 39771 geometries with energies up to $20000 \mathrm{~cm}^{-1}$ above the global minima was generated for the purpose of fitting the 9-D potential surface. The geometries were selected by randomly sampling the internal coordinates. Three separate sampling schemes were used to generate sets of points designed to capture the behaviour of the potential in the bonded, roaming and dissociated regimes. 
TABLE I: Parameters of $C_{s}$ and $C_{2 v}$ structures and optimised with fc-CCSD(T) $\left(\mathrm{F} 12^{*}\right)$ using augcc-pVXZ basis sets $(\mathrm{X}=\mathrm{D}, \mathrm{T}, \mathrm{Q})$. Bond lengths are in bohr and angles in degrees and dissociation energies in $\mathrm{cm}^{-1}$. Corresponding parameters for the analytic 9-D surface are also tabulated.

\begin{tabular}{llllll}
\hline \hline & & $\mathrm{DZ}$ & $\mathrm{TZ}$ & $\mathrm{QZ}$ & $\mathrm{PES}$ \\
\hline$C_{s}$ & $R_{0}$ & 4.9902 & 4.9990 & 4.9986 & 5.0067 \\
& $R_{1 A}$ & 1.8135 & 1.8128 & 1.8117 & 1.8126 \\
& $R_{1 B}$ & 1.7645 & 1.7641 & 1.7633 & 1.7637 \\
& $\theta_{A}$ & 105.43 & 105.44 & 105.50 & 105.46 \\
& $\beta_{A}$ & 46.33 & 46.14 & 46.02 & 46.38 \\
& $\beta_{B}$ & 2.50 & 2.53 & 2.51 & 2.69 \\
& $D_{e}$ & 3122.7 & 3074.0 & 3062.9 & 3005.5 \\
$C_{2 v}$ & $R_{0}$ & 5.0460 & 5.0514 & 5.0491 & 5.0568 \\
& $R_{1 A}$ & 1.8104 & 1.8096 & 1.8092 & 1.8096 \\
& $R_{1 B}$ & 1.7596 & 1.7595 & 1.7589 & 1.7593 \\
& $\theta_{A}$ & 106.45 & 106.42 & 106.45 & 106.43 \\
\hline \hline
\end{tabular}

The first set, accounting for approximately half of the geometries, was constructed to sample the bound region of the potential, including the $C_{2 v}$ and $C_{s}$ stationary points. First, the ranges of internal coordinates within the $20000 \mathrm{~cm}^{-1}$ target window were determined through 1-D scans of the potential. Then, displacements of internal coordinates were generated by randomly sampling from a distribution within that range, weighted towards low energies, using the same approach as in Ref. [38].

The second set, accounting for approximately a quarter of the data points, was constructed to ensure the potential dissociates into the sum of the potentials for $\mathrm{H}_{2} \mathrm{O}$ and $\mathrm{HF}$. Here the four internal coordinates of the fragments were displaced in a manner analogous to that for the first set, but where the reference structure was the equilibrium geometry of the fragments at $20 \AA$ separation.

The third set was constructed to sample the roaming region of the potential. $\mathrm{H}_{2} \mathrm{O}$ and $\mathrm{HF}$ geometries with small random displacements of their internal coordinates at the $C_{2 v}$ equilib- 
TABLE II: Harmonic wavenumbers at $C_{s}$ and $C_{2 v}$ stationary points on fc-CCSD $(\mathrm{T})\left(\mathrm{F} 12^{*}\right) /$ aug-ccpVXZ $(\mathrm{X}=\mathrm{D}, \mathrm{T}, \mathrm{Q})$ potential energy surfaces and the analytic 9-D potential.

\begin{tabular}{|c|c|c|c|c|c|c|}
\hline & Mode & Sym. & $\mathrm{DZ}$ & $\mathrm{TZ}$ & $\mathrm{QZ}$ & PES \\
\hline \multirow[t]{9}{*}{$C_{s}$} & 1 & $a^{\prime}$ & 278 & 240 & 197 & 219 \\
\hline & 2 & $a^{\prime \prime}$ & 238 & 226 & 234 & 237 \\
\hline & 3 & $a^{\prime}$ & 336 & 265 & 248 & 263 \\
\hline & 4 & $a^{\prime}$ & 721 & 707 & 694 & 695 \\
\hline & 5 & $a^{\prime \prime}$ & 844 & 825 & 824 & 823 \\
\hline & 6 & $a^{\prime}$ & 1639 & 1653 & 1645 & 1650 \\
\hline & 7 & $a^{\prime}$ & 3823 & 3786 & 3785 & 3796 \\
\hline & 8 & $a^{\prime}$ & 3846 & 3837 & 3836 & 3840 \\
\hline & 9 & $a^{\prime \prime}$ & 3940 & 3938 & 3944 & 3940 \\
\hline \multirow[t]{9}{*}{$C_{2 v}$} & 1 & $b_{2}$ & $207 i$ & $215 i$ & $203 i$ & $204 i$ \\
\hline & 2 & $b_{1}$ & 205 & 205 & 205 & 201 \\
\hline & 3 & $a_{1}$ & 232 & 229 & 230 & 228 \\
\hline & 4 & $b_{2}$ & 650 & 620 & 620 & 620 \\
\hline & 5 & $b_{1}$ & 787 & 773 & 770 & 763 \\
\hline & 6 & $a_{1}$ & 1646 & 1648 & 1647 & 1647 \\
\hline & 7 & $a_{1}$ & 3850 & 3845 & 3844 & 3851 \\
\hline & 8 & $a_{1}$ & 3861 & 3856 & 3854 & 3858 \\
\hline & 9 & $b_{1}$ & 3965 & 3963 & 3961 & 3963 \\
\hline
\end{tabular}

rium were randomly oriented with respect to each other with intermolecular $\mathrm{OH}$ distances in the range $1.5-3.5 \AA$.

\section{9-D analytic potential energy fit}

The strategy we followed for obtaining an analytic 9-D potential energy surface that closely reproduces the ab initio data is to first construct a simple model that captures the 
TABLE III: Single point energy differences in $\mathrm{cm}^{-1}$ at the fc-CCSD(T)(F12*)/aug-cc-pVTZ $C_{s}$ and $C_{2 v}$ stationary points at different basis sets, with counter-poise correction (CC), and with corrections for core-valence (CV) and high order correlation effects T, (Q).

\begin{tabular}{lll}
\hline \hline Method & Basis & Energy \\
\hline fc-CCSD $(\mathrm{T})\left(\mathrm{F} 12^{*}\right)$ & aug-cc-pVTZ & 126.6 \\
fc-CCSD $(\mathrm{T})\left(\mathrm{F} 12^{*}\right)$ & aug-cc-pVQZ & 113.9 \\
fc-CCSD $(\mathrm{T})\left(\mathrm{F} 12^{*}\right)$ & aug-cc-pV5Z & 113.3 \\
fc-CCSD $(\mathrm{T})\left(\mathrm{F} 12^{*}\right)$ & aug-cc-pwCVQZ & 113.7 \\
CC fc-CCSD $(\mathrm{T})\left(\mathrm{F} 12^{*}\right)$ & aug-cc-pVTZ & 108.6 \\
CC fc-CCSD $(\mathrm{T})\left(\mathrm{F} 12^{*}\right)$ & aug-cc-pVQZ & 112.0 \\
CC fc-CCSD $(\mathrm{T})\left(\mathrm{F} 12^{*}\right)$ & aug-cc-pV5Z & 112.7 \\
CC fc-CCSD $(\mathrm{T})\left(\mathrm{F} 12^{*}\right)$ & aug-cc-pV5Z & 112.7 \\
$+\mathrm{CV}\left[\mathrm{CCSD}(\mathrm{T})\left(\mathrm{F} 12^{*}\right)\right]$ & aug-cc-pwCVQZ & 106.9 \\
$+\mathrm{T}-(\mathrm{T})[\mathrm{fc}-\mathrm{CCSDT}]$ & cc-pVTZ & 106.5 \\
+ (Q) $[\mathrm{fc}-\mathrm{CCSDT}(\mathrm{Q})]$ & cc-pVTZ & 109.3 \\
\hline \hline
\end{tabular}

broad features of the potential, and then to fit a correction surface to recover the finer details. A similar approach has been found to be effective in fitting compact and accurate analytic potentials for malonaldehye,[38] formic acid,[39, 40] and metaphosphosous acid.[41]

The form of the zeroth-order model potential was chosen to be

$$
\begin{aligned}
V_{0}(\mathbf{r})= & C+V_{1}^{\mathrm{MO}}\left(r_{O_{1}}\right)+V_{1}^{\mathrm{MO}}\left(r_{O H_{2}}\right)+V_{2}^{\mathrm{MO}}\left(r_{O H_{3}}\right)+V_{3}^{\mathrm{MO}}\left(r_{F H_{3}}\right) \\
& +V_{1}^{\mathrm{Q}}\left(r_{O_{3}}\right)+V_{2}^{\mathrm{Q}}\left(r_{H_{1} H_{3}}\right)+V_{2}^{\mathrm{Q}}\left(r_{H_{2} H_{3}}\right)+V_{3}^{\mathrm{Q}}\left(r_{O F}\right)+V_{4}^{\mathrm{Q}}\left(r_{F H_{1}}\right)+V_{4}^{\mathrm{Q}}\left(r_{F H_{2}}\right)
\end{aligned}
$$

with

$$
\begin{aligned}
V^{\mathrm{MO}}(r) & =D^{2}\left(1-\exp \left(-\alpha\left(r-r_{e}\right)\right)\right)^{2} \\
V^{Q}(r) & =q_{1} q_{2} / r+A^{2} \exp \left(\gamma\left(r_{x}-r\right)\right)
\end{aligned}
$$

The atom labelling is such that hydrogens 1 and 2 are bonded to the $\mathrm{O}$ atom and hydrogen 3 is bonded to the $\mathrm{F}$ atom and $r_{\mathrm{OH}_{1}}$ is the distance between the oxygen and hydrogen 1, 
and so on. The potential is manifestly symmetric with respect to exchange of the $\mathrm{H}$ atoms of the water moiety. The constant $C$, the three sets of Morse parameters $D, \alpha, r_{e}$ and four sets of electrostatic and exchange repulsion parameters $q_{1}, q_{2}, A, \gamma$ and $r_{x}$ were fit to the data points. Steepest descent optimisation of the root mean squared deviations (RMSD) was applied starting from parameters fit to ab-initio data along 1-D cuts of the potential.

Following earlier work, $[38,39]$ the correction surface was chosen to be a sum of multivariate Gaussians of atom-atom separations, with centres at grid points distributed over the coordinate ranges relevant to the $0-20000 \mathrm{~cm}^{-1}$ energy window. The form of the final potential is

$$
\begin{aligned}
V(\mathbf{r}) & =V_{0}(\mathbf{r})+\sum_{i} \sum_{\mu_{i}} d_{i}^{\mu}\left(e^{-\beta_{i}^{\mu}\left(r_{i}-c_{i}^{\mu}\right)^{2}}-s_{i}^{\mu}\right) \\
& +\sum_{i>j} \sum_{\nu_{i j}} d_{i j}^{\nu}\left(e^{-\beta_{i}^{\nu}\left(r_{i}-c_{i}^{\nu}\right)^{2}-\beta_{j}^{\nu}\left(r_{j}-c_{j}^{\nu}\right)^{2}}-s_{i j}^{\nu}\right) \\
& +\cdots
\end{aligned}
$$

with up to 4-body Gaussians included. The index $\mu$ labels a Gaussian centred at $c_{i}^{\mu}$ with width parameter $\beta_{i}^{\mu}$. The corresponding shift parameter $s_{i}^{\mu}$ is fixed by imposing that functions are standardised against the data for the fitting procedure. The non-linear parameters were chosen such that the overall basis was composed of three sets of 1-D, 2-D, 3-D and 4-D grids of functions: a widely spaced grid of diffuse Gaussians to capture slowly varying features, a medium grid, and a finer grid of compact Gaussians to capture the finer features of the correction surface. The coefficients $d_{i}^{\mu}$ of the resulting distributed Gaussian basis were fit using the LASSO (least absolute shrinkage and selection operator) method,[42] which selects only those functions from the set that have the largest effect on reducing the least squares deviation from the ab initio data. Data was fit using weights $w_{i}=100 /\left(E_{i}+100\right)$ to ensure that the cumulative RMSD grows approximately linearly as a function of the energy $E_{i}$ above the reference, in $\mathrm{cm}^{-1}$. The LASSO optimisation was stopped after the RMSD dropped below $0.15 \%$ over the $10000 \mathrm{~cm}^{-1}$ range. The quality of the fit for the resulting potential is displayed in Fig 2 and the structures of the $C_{2 v}$ and $C_{s}$ stationary points on the analytic fit are listed in Table I. The corresponding harmonic wavenumbers of the potential are listed in Table II. 

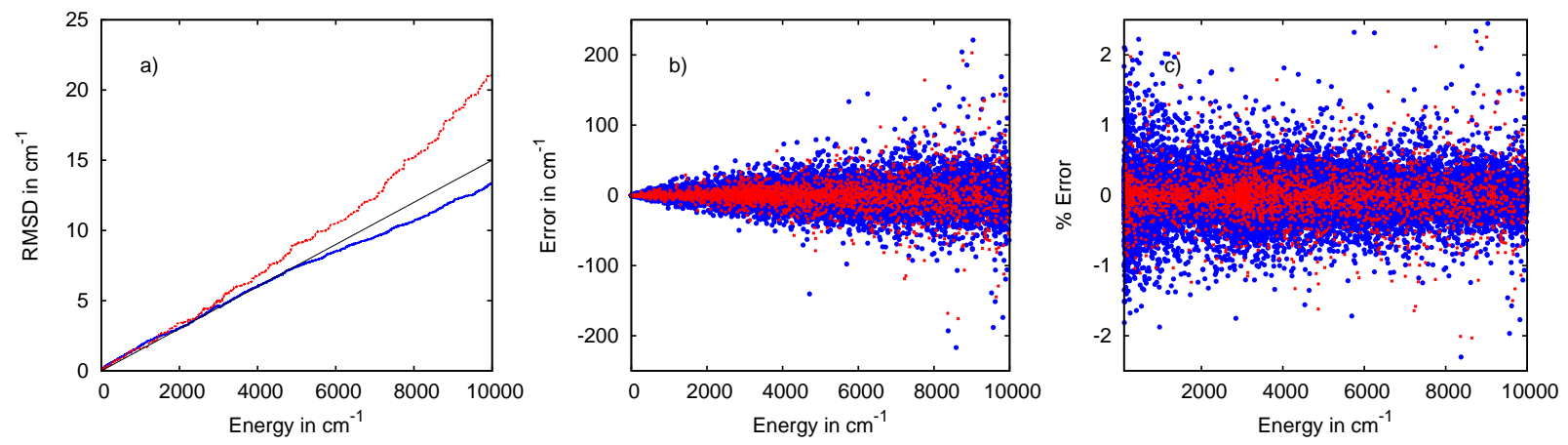

FIG. 2: a) Cumulative root mean squared deviation of the analytic potential from the ab initio data. b) Scatter plot of errors. c) Scatter plot of percentage errors. Blue points are from the training set and red points are from the control set.

\section{PROPERTIES OF THE POTENTIAL ENERGY SURFACE}

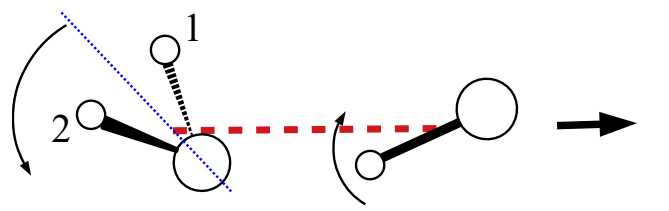

$(180,48,90 ; 0,3)$

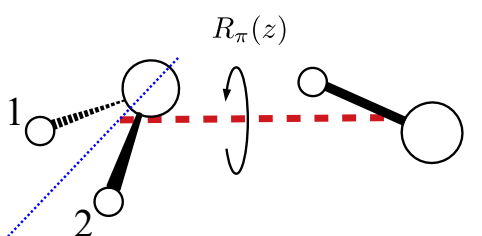

$(0,48,270 ; 180,3)$

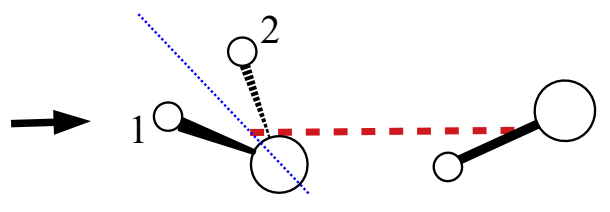

$(180,48,270 ; 0,3)$

$X$

FIG. 3: The tunnelling path from $\mathrm{X}$ to $\mathrm{Y}$. $\mathrm{Y}^{\prime}$ is obtained from $\mathrm{Y}$ by a rotation of the whole molecule, $R_{\pi}(z)$. The dashed $\mathrm{OH}$ bond is behind the plane containing HF and $\mathrm{O}$. The red dashed line connects the centers of mass of the monomers. In brackets are the coordinates $\left(\alpha_{A}, \beta_{A}, \gamma_{A}, \alpha_{B}, \beta_{B}\right)$.

The 9-D PES is converted to a 5-D PES, which we use in our calculations, by evaluating it at fixed values of the intramolecular coordinates. The fixed values for $\mathrm{H}_{2} \mathrm{O}$ and $\mathrm{HF}$ were computed from ab initio PESs. For the water molecule, we used the ground state values $\langle r\rangle(\mathrm{O}-\mathrm{H})=1.8437015$ Bohr and $\theta(\mathrm{H}-\mathrm{O}-\mathrm{H})=104.430^{\circ}$, given in Ref. [43] and in Table 2 of Ref. [44]. For HF, we use the ground state value $\langle r\rangle=r(\mathrm{H}-\mathrm{F})=1.76286$ Bohr (from Table IV. of Ref. [45]). The stationary points of the $\mathrm{H}_{2} \mathrm{O}-\mathrm{HF}$ are reported in Table IV. The energy at the minimum of the 5-D PES is $109.955 \mathrm{~cm}^{-1}$ and the saddle point is 252.036 
TABLE IV: $\mathrm{H}_{2} \mathrm{O}-\mathrm{HF}$ stationary points on the 5-D PES. Distances are in bohr and angles are in degrees. Monomer $\mathrm{A}$ is $\mathrm{H}_{2} \mathrm{O}$ and $\mathrm{B}$ is $\mathrm{HF}$. The coordinates of $\mathrm{H}_{2} \mathrm{O}$ are bond coordinates. $V$ values are with respect to the minimum of the 9-D PES. On our 5-D PES $D_{e}=3072.55 \mathrm{~cm}^{-1}$.

\begin{tabular}{lll}
\hline \hline & Minimum & Saddle point \\
\hline$\left(\alpha_{A}, \beta_{A}, \gamma_{A}\right)$ & $(180,48.672,90)$ & $(0,0,0)$ \\
$\left(\alpha_{B}, \beta_{B}\right)$ & $(0,2.849)$ & $(0,0)$ \\
$R_{0}$ & 5.005 & 5.067 \\
$\left(R_{1 A}, R_{2 A}, \theta_{A}\right)$ & $(1.844,1.844,104.43)$ & $(1.844,1.844,104.43)$ \\
$R_{1 B}$ & 1.76286 & 1.76286 \\
& & \\
$\mathrm{~V}\left(\mathrm{~cm}^{-1}\right)$ & 109.955 & 252.036 \\
\hline \hline
\end{tabular}

$\mathrm{cm}^{-1}$, corresponding to a barrier height of $142.081 \mathrm{~cm}^{-1}$. The geometry of one of the global minima $\left(C_{s}\right.$ symmetry) is shown in Fig. 1 and also in Fig. 3 (as $\mathrm{X}$ on the left). At the global minimum, the hydrogen bond is not linear : if the $\mathrm{O}$ atom points downwards, $\mathrm{H}$ of HF points slightly downwards. X, on the left in figure 3, and $\mathrm{Y}$, in the middle of figure 3 , are two equivalent versions; $\mathrm{Y}=E^{*} \mathrm{X}$, where

$$
E^{*} f\left(\alpha_{A}, \beta_{A}, \gamma_{A} ; \alpha_{B}, \beta_{B} ; \alpha, \beta\right)=f\left(\pi-\alpha_{A}, \beta_{A}, 2 \pi-\gamma_{A} ; \pi-\alpha_{B}, \beta_{B} ; \pi+\alpha, \pi-\beta\right)
$$

There is a tunnelling path between $\mathrm{X}$ and $\mathrm{Y}$. Along the path $\mathrm{H}_{2} \mathrm{O}$ wags, which changes $\beta_{A}$, and the $\mathrm{H}$ of HF moves upward, changing $\beta_{B}$. Y and $\mathrm{Y}^{\prime}$ are the same point on the PES. A rotation of $\pi$ about $\mathbf{r}_{\mathbf{0}}$ links $\mathrm{Y}$ to $\mathrm{Y}^{\prime}$. (12) $\mathrm{X}=\mathrm{Y}^{\prime}$. Because $\mathrm{Y}$ and $\mathrm{Y}^{\prime}$ are obtained by applying $E^{*}$ and (12) to X, the symmetry of the lowest upper tunnelling level must be $B^{-}$ where $B$ indicates that a state is anti-symmetric with respect to the permutation of the two $\mathrm{H}$ in the water molecule and - denotes the parity. More rigorously, to obtain irrep labels of the states split by tunnelling, one follows Dyke [46] and decomposes $A^{\prime}$, the $C_{s}$ irrep of the ground state, into the irreps of $G_{4}$. This yields $A^{\prime}\left(C_{s}\right)=A^{+}+B^{-}$. The tunnelling path is basically the same as the $\nu_{\beta(o)}\left(B^{-}\right)$normal coordinate. [7]. It is similar to the acceptor switch tunneling path of $\left(\mathrm{H}_{2} \mathrm{O}\right)_{2}$ (see Fig. 3 of [47]). 


\section{CALCULATIONAL DETAILS}

The computational procedure is similar to that used in many papers. $[18,19,19,48-$ 50] Eigenvalues and eigenvectors are computed with the Lanczos algorithm and about 4000 iterations. Quadrature is used for potential matrix elements and matrix-vector products are evaluated by doing sums sequentially. We have done calculations with different basis sizes to test convergence. The final bend basis set has $j_{\max }=15$ for both $\mathrm{H}_{2} \mathrm{O}$ and $\mathrm{HF}$ and the potential integrals are computed with 16 Gauss-Legendre quadrature points for $\beta_{A}$ and $\beta_{B}$ coordinates and 32 evenly spaced quadrature points in $[0,2 \pi]$ range for $\gamma_{A}$ and $\alpha_{A}-\alpha_{B}$ coordinates. For the stretch basis, we use $N_{r_{0}}=120$ sine DVR functions in the range $[4,11]$ bohr. By comparing with results obtained from smaller bases, it was established that levels we report are converged to $0.008 \mathrm{~cm}^{-1}$.

The rotational constants required in the $\mathrm{KEO}$ are ground vibrational state experimental values: $\mathrm{H}_{2} \mathrm{O}\left(\mathrm{A}=27.8806 \mathrm{~cm}^{-1}, \mathrm{~B}=9.2778 \mathrm{~cm}^{-1}\right.$, and $\left.\mathrm{C}=14.5216 \mathrm{~cm}^{-1}\right)[51]$ and $\mathrm{HF}(\mathrm{B}$ $\left.=20.5567 \mathrm{~cm}^{-1}\right)[52]$. The atomic masses used are: $\mathrm{H}(1.007825 \mathrm{u}),{ }^{16} \mathrm{O}(15.994915 \mathrm{u})$, and ${ }^{19} \mathrm{~F}(18.9984 \mathrm{u})[53]$.

We use the NNR code, where NNR stands for nonlinear-nonlinear-rigid monomers.[54] To evaluate the PES at our quadrature points we transform from the Euler angles we use to Cartesian coordinates in the DF frame, i.e. we need $X^{D F}=X^{D F}\left(\alpha_{A}-\alpha_{B}, \beta_{A}, \gamma_{A}, \beta_{B}, r_{0}\right)$ for each atom $i$. We use

$$
\begin{aligned}
& \mathbf{X}_{\mathbf{A} \mathbf{i}}^{\mathbf{D F}}=\mathbf{S}^{\mathbf{t}}\left(\alpha_{A}, \beta_{A}, \gamma_{A}\right) \mathbf{x}_{\mathbf{A} \mathbf{i}}^{\mathbf{M F}}, \\
& \mathbf{X}_{\mathbf{B i}}^{\mathbf{D F}}=\mathbf{S}^{\mathbf{t}}\left(\alpha_{B}, \beta_{B}, 0\right) \mathbf{x}_{\mathbf{B i}}^{\mathbf{M F}}+\left(0,0, r_{0}\right)^{t},
\end{aligned}
$$

where $\mathbf{S}$ is the direction cosine matrix defined in Ref. [23]. The elements of $x_{A / B i}^{M F}$ are the Cartesian coordinates of atom $i$ in the MF frame.

\section{A. Intensity calculations}

Computing intensities enables us to confirm assignments of Kisiel et al. $[7,8]$ and identify new bands, which should be observable in microwave spectra. Intensities are calculated from 

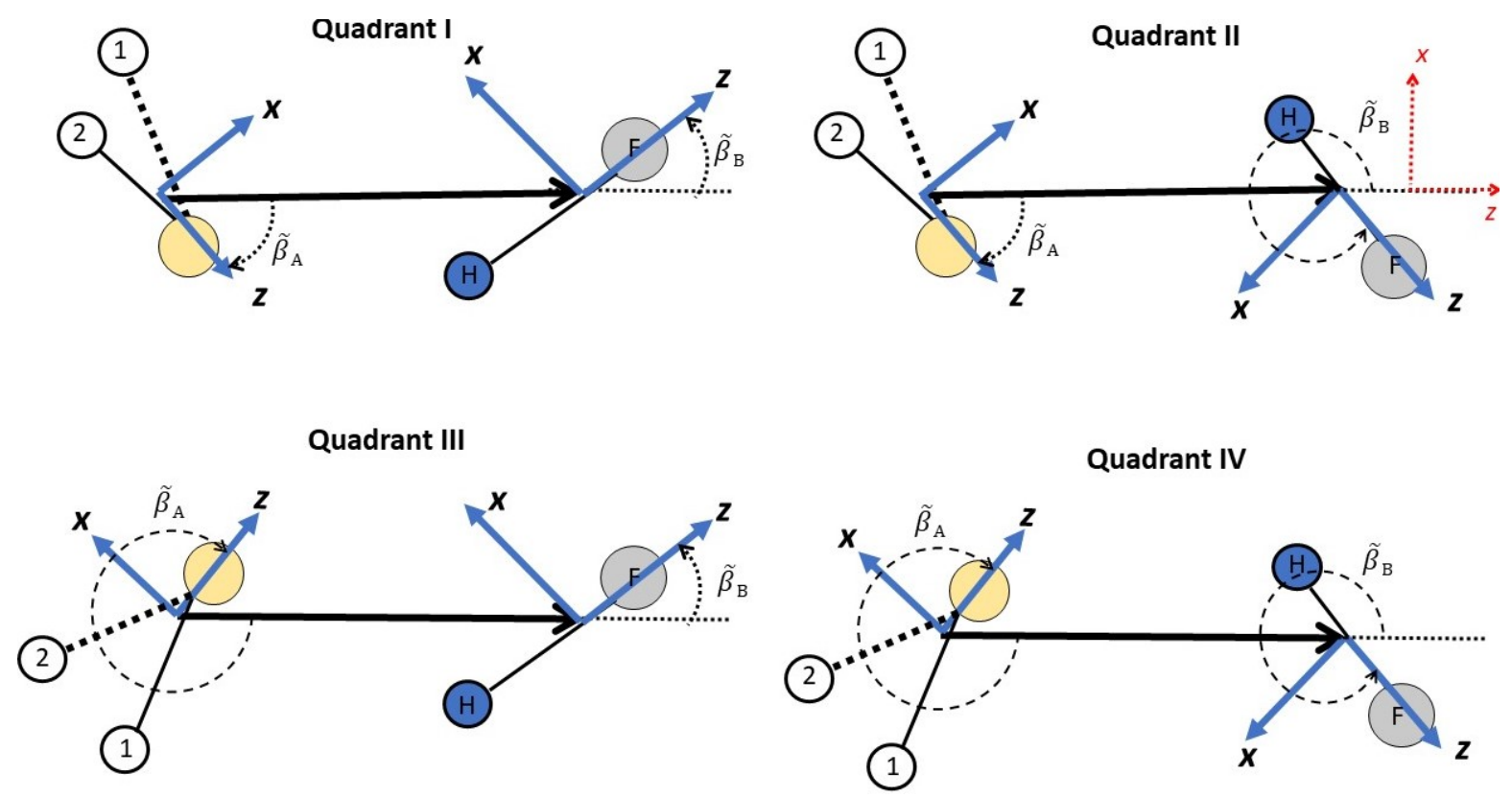

FIG. 4: Definition of the extended angles $\tilde{\beta_{A}}, \tilde{\beta_{B}}$ used for visualizing wavefunctions of $\mathrm{H}_{2} \mathrm{O}-\mathrm{HF}$.

eigenvectors. The line strength is defined as

$$
S_{i^{\prime} i}=3\left|\sum_{M, M^{\prime}}\left\langle\Psi_{i^{\prime}}\left|\mu_{0}^{\mathrm{SF}}\right| \Psi_{i}\right\rangle\right|^{2},
$$

where $\mu_{0}^{\mathrm{SF}}=\mu_{Z}^{\mathrm{SF}}$ is the space-fixed $Z$ component of the dipole moment operator and $\Psi_{i}$ is a rovibrational wavefunction [55]. Because it is easiest to calculate the integral when $\Psi_{i}$ is a sum of primitive basis functions, $\Psi_{i}$, computed in the parity-adapted basis, is re-written as a linear combination of primitive basis functions in Eq. 1. To compute line strengths one then needs matrix elements [23, 55],

$$
\begin{aligned}
& \left\langle j_{A}^{\prime} k_{A}^{\prime} m_{A}^{\prime} ; j_{B}^{\prime} m_{B}^{\prime} ; J^{\prime} K^{\prime} M^{\prime}\left|\mu_{0}^{\mathrm{SF}}\right| j_{A} k_{A} m_{A} ; j_{B} m_{B} ; J K M\right\rangle \\
& =\mu_{\mathrm{H}_{2} \mathrm{O}} \delta_{j_{B}^{\prime}, j_{B}} \delta_{m_{B}^{\prime}, m_{B}} \sum_{\sigma=-1}^{1}(-1)^{k_{A}^{\prime}+m_{A}^{\prime}}\left[j_{A}^{\prime}\right]\left[j_{A}\right]\left(\begin{array}{ccc}
j_{A}^{\prime} & 1 & j_{A} \\
-k_{A}^{\prime} & 0 & k_{A}
\end{array}\right)\left(\begin{array}{ccc}
j_{A}^{\prime} & 1 & j_{A} \\
-m_{A}^{\prime} & \sigma & m_{A}
\end{array}\right) \\
& \times(-1)^{K^{\prime}+M^{\prime}}\left[J^{\prime}\right][J]\left(\begin{array}{ccc}
J^{\prime} & 1 & J \\
-K^{\prime} & \sigma & K
\end{array}\right)\left(\begin{array}{ccc}
J^{\prime} & 1 & J \\
-M^{\prime} & 0 & M
\end{array}\right)
\end{aligned}
$$

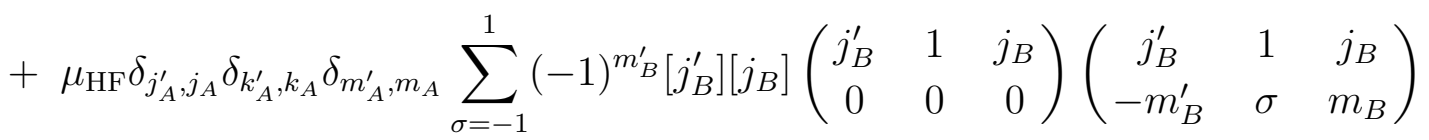

$$
\begin{aligned}
& \times(-1)^{K^{\prime}+M^{\prime}}\left[J^{\prime}\right][J]\left(\begin{array}{ccc}
J^{\prime} & 1 & J \\
-K^{\prime} & \sigma & K
\end{array}\right)\left(\begin{array}{ccc}
J^{\prime} & 1 & J \\
-M^{\prime} & 0 & M
\end{array}\right),
\end{aligned}
$$


TABLE V: $A^{+}$and $B^{-}$vibrational levels $\left(\right.$in $\mathrm{cm}^{-1}$ ) computed using the basis defined by $j_{1 x}=$ $j_{2 x}=15, n_{r_{0}}=120$, and $r_{0} \in[4,11]$ bohr. The vibrational levels are relative to the zero-point energy (ZPE) at $1080.166 \mathrm{~cm}^{-1}$.

\begin{tabular}{|c|c|c|c|}
\hline Assignment & $A^{+}$ & Assignment & $B^{-}$ \\
\hline$G S$ & 0.000 & $\nu_{\beta(o)}$ & 81.862 \\
\hline$\nu_{\sigma}$ & 195.997 & $\nu_{\sigma}+\nu_{\beta(o)}$ & 281.782 \\
\hline $2 \nu_{\beta(o)}$ & 238.228 & $3 \nu_{\beta(o)}$ & 394.781 \\
\hline $2 \nu_{\sigma}$ & 381.931 & $2 \nu_{\sigma}+\nu_{\beta(o)}$ & 470.856 \\
\hline $2 \nu_{\beta(i)}$ & 385.970 & $\nu_{\beta(o)}+2 \nu_{\beta(i)}$ & 511.467 \\
\hline$\nu_{\sigma}+2 \nu_{\beta(o)}$ & 436.626 & $\nu_{B(o)}$ & 577.164 \\
\hline $3 \nu_{\sigma}$ & 558.902 & $\nu_{\sigma}+3 \nu_{\beta(o)}$ & 590.151 \\
\hline $4 \nu_{\beta(o)}$ & 563.212 & $3 \nu_{\sigma}+\nu_{\beta(o)}$ & 649.258 \\
\hline$\nu_{\sigma}+2 \nu_{\beta(i)}$ & 575.441 & $\nu_{\sigma}+\nu_{\beta(o)}+2 \nu_{\beta(i)}$ & 703.200 \\
\hline $2 \nu_{\beta(o)}+2 \nu_{\sigma}$ & 623.366 & $5 \nu_{\beta(o)}$ & 733.010 \\
\hline $2 \nu_{\beta(o)}+2 \nu_{\beta(i)}$ & 675.914 & $\nu_{\sigma}+\nu_{B(o)}$ & 757.782 \\
\hline$\nu_{\beta(o)}+\nu_{B(o)}$ & 692.101 & $2 \nu_{\sigma}+3 \nu_{\beta(o)}$ & 774.294 \\
\hline $4 \nu_{\sigma}$ & 725.752 & $4 \nu_{\sigma}+\nu_{\beta(o)}$ & 817.293 \\
\hline $4 \nu_{\beta(i)}$ & 746.954 & $3 \nu_{\beta(o)}+2 \nu_{\beta(i)}$ & 838.722 \\
\hline $2 \nu_{\sigma}+2 \nu_{\beta(i)}$ & 754.262 & $\cdots$ & \\
\hline$\nu_{\sigma}+4 \nu_{\beta(o)}$ & 755.890 & $2 \nu_{\sigma}+\nu_{\beta(o)}+2 \nu_{\beta(i)}$ & 883.646 \\
\hline $3 \nu_{\sigma}+2 \nu_{\beta(o)}$ & 799.049 & $\nu_{\sigma}+5 \nu_{\beta(o)}$ & 916.993 \\
\hline$\nu_{\sigma}+2 \nu_{\beta(o)}+2 \nu_{\beta(i)}$ & 860.257 & $\nu_{\beta(o)}+4 \nu_{\beta(i)}$ & 935.002 \\
\hline$\nu_{\sigma}+\nu_{\beta(o)}+\nu_{B(o)}$ & 873.641 & $3 \nu_{\sigma}+3 \nu_{\beta(o)}$ & 947.107 \\
\hline $5 \nu_{\sigma}$ & 881.724 & $5 \nu_{\sigma}+\nu_{\beta(o)}$ & 975.623 \\
\hline $6 \nu_{\beta(o)}$ & 902.939 & $\cdots$ & \\
\hline $3 \nu_{\sigma}+2 \nu_{\beta(i)}$ & 920.450 & $\nu_{\sigma}+3 \nu_{\beta(o)}+2 \nu_{\beta(i)}$ & 1021.792 \\
\hline$\nu_{\sigma}+4 \nu_{\beta(i)}$ & 933.625 & $3 \nu_{\sigma}+\nu_{\beta(o)}+2 \nu_{\beta(i)}$ & 1053.281 \\
\hline
\end{tabular}


where $[J]=\sqrt{2 J+1}$. Finally, when computing the line strength, the summation over $M$ and $M^{\prime}$ in Eq. (8) can be factored out and evaluated explicitly [56],

$$
\sum_{M, M^{\prime}}\left(\begin{array}{ccc}
J^{\prime} & 1 & J \\
-M^{\prime} & 0 & M
\end{array}\right)^{2}=\frac{1}{3}
$$

The NNR code computes $\langle\mu\rangle=\left\langle\Psi_{i^{\prime}}\left|\mu_{0}^{\mathrm{SF}}\right| \Psi_{i}\right\rangle /\left(\begin{array}{ccc}J^{\prime} & 1 & J \\ -M^{\prime} & 0 & M\end{array}\right) /(-1)^{M^{\prime}}$, which is independent of $M$ and $M^{\prime}$. The line strength is simply $S_{i^{\prime} i}=|\langle\mu\rangle|^{2}$ due to Eq. (10). To determine intensities from the line strengths one must multiply by the appropriate Boltzmann factor and also by nuclear statistical weights. The weights are important as they enhance the intensities of transitions from states with larger $g$ values: $g_{A^{+} / A^{-}}=1$ and $g_{B^{+} / B^{-}}=3$. We consider that the dipole moment of the complex is the sum of the dipole moments of $\mathrm{H}_{2} \mathrm{O}$ and of $\mathrm{HF}$ and use vibrationally averaged permanent dipole values: for $\mu_{\mathrm{H}_{2} \mathrm{O}}=0.7277 \mathrm{e} a_{0}[57]$ and for $\mu_{H F}=0.7083 \mathrm{e} a_{0}[58]$.

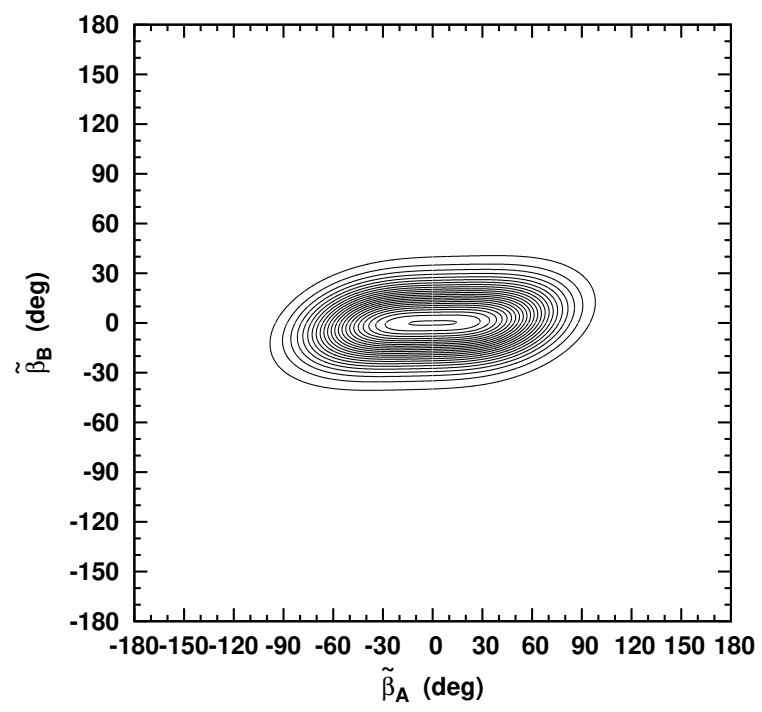

FIG. 5: Wavefunction cut $\left(\tilde{\beta_{A}}, \tilde{\beta_{B}}\right)$ for the $A^{+}$state at $0.0 \mathrm{~cm}^{-1}$.

\section{RESULTS AND DISCUSSION}

\section{A. Assignment of vibrational states}

Assigned vibrational energy levels are presented in tables V and VI. This is the first time that many vibrational states have been computed from a 5-D intermolecular Hamiltonian. 
The most important tool we use to assign levels is wavefunction plots. The coordinates not shown in the wavefunction plots are fixed at equilibrium values.
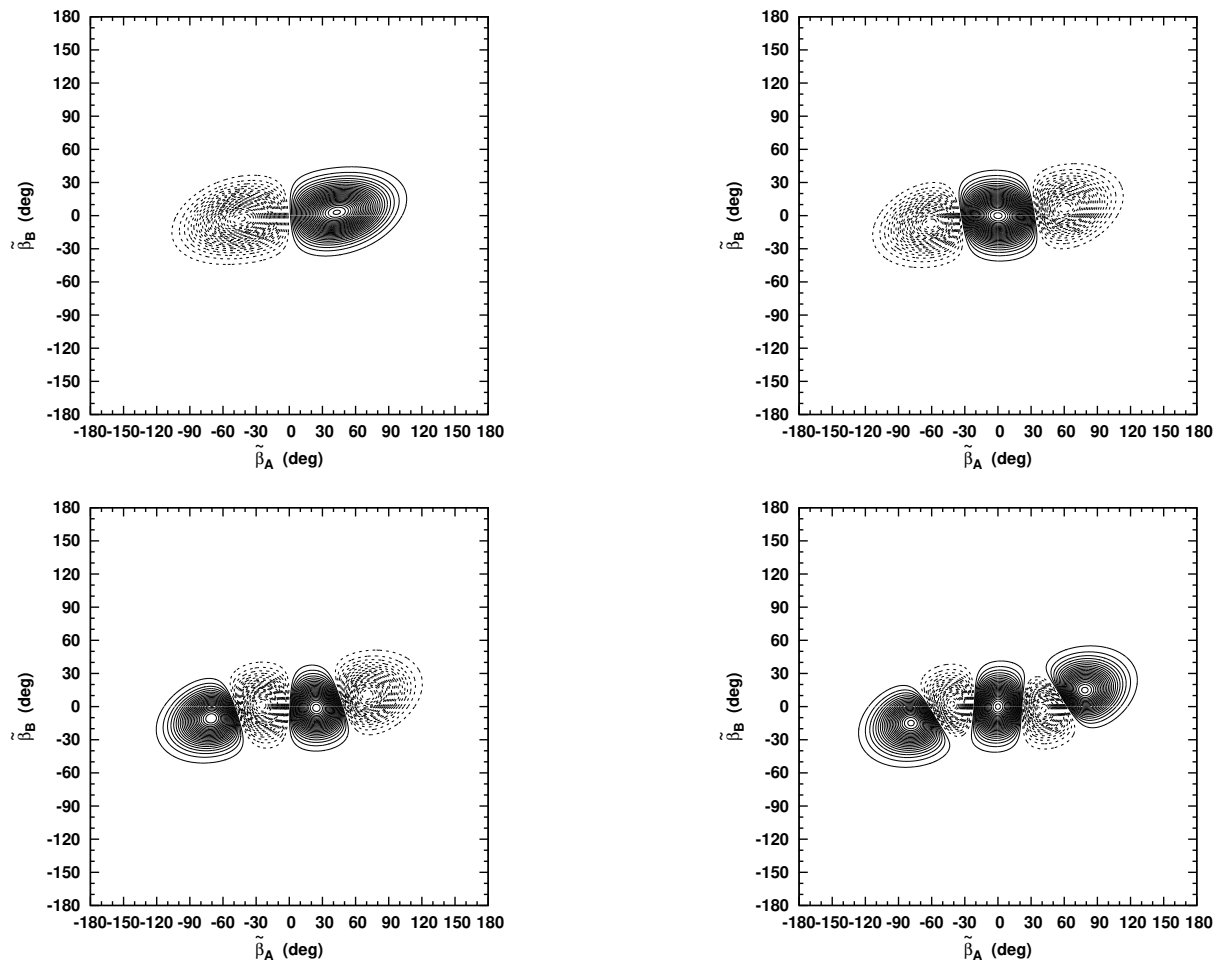

FIG. 6: Wavefunction cut $\left(\tilde{\beta_{A}}, \tilde{\beta_{B}}\right)$ plots for the $\nu_{\beta(o)}\left(B^{-}\right)$state (top left) and its overtones $2 \nu_{\beta(o)}\left(A^{+}\right)$(top right), $3 \nu_{\beta(o)}\left(B^{-}\right)$(bottom left), $4 \nu_{\beta(o)}\left(A^{+}\right)$(bottom right).
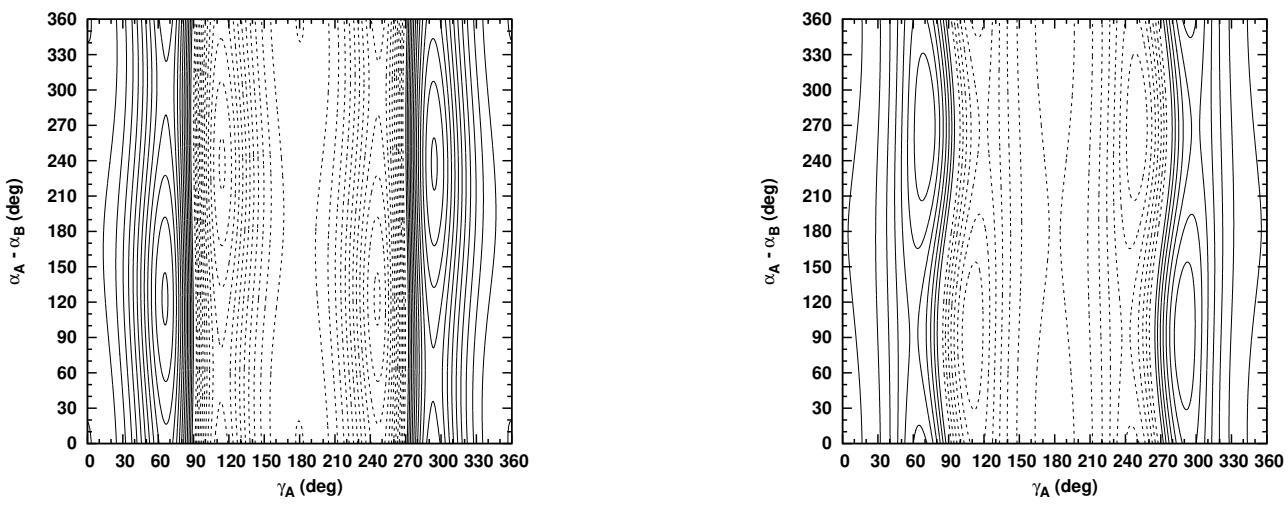

FIG. 7: Wavefunction cut $\left(\alpha_{A}-\alpha_{B}, \gamma_{A}\right)$ plots for the $\nu_{\beta(i)}\left(B^{+}\right)$state whose energy is 196.131 $\mathrm{cm}^{-1}$ (on the left) and the $\nu_{B(i)}$ state at $679.791 \mathrm{~cm}^{-1}$ (on the right).

We plot in extended coordinates $\tilde{\beta_{A}}, \tilde{\beta_{B}}$, defined in the range $[-\pi, \pi]$, because they make 
it possible to more easily visualize wavefunctions. Similar coordinates were used for $\mathrm{H}_{2} \mathrm{O}-$ $\mathrm{N}_{2}$ dimer in Ref. [18]. To plot as a function of $\tilde{\beta_{A}}, \tilde{\beta_{B}}$, we need relationships between the extended coordinates in all four quadrants and the dynamical coordinates $\beta_{A}, \beta_{B}$. They are illustrated in Figure 4.

Figure 5 displays the ground state wavefunction in extended $\tilde{\beta}_{k}$ coordinates. The maximum of the wavefunction is at the top of a barrier $\left(\tilde{\beta}_{A}=0^{\circ}\right)$ and the wavefunction has significant amplitude in both wells.

Fig. 6 shows states with excitation in the inversion coordinate which is $\nu_{\beta(o)}$. The $\nu_{\beta(o)}$ state (top left) has two lobes (the negative lobe has dashed contours) that are well separated along $\tilde{\beta_{A}}$. $\beta_{A}$ is essentially the tunnelling coordinate and we expect one node because $\nu_{\beta(o)}$ is a $\mathrm{B}$ state and anti-symmetric with respect to $\mathrm{H}-\mathrm{H}$ exchange in the water molecule. Fig. 6 is similar to Fig. 8 of Loreau et al.'s paper [20] where polyspherical angles are used.
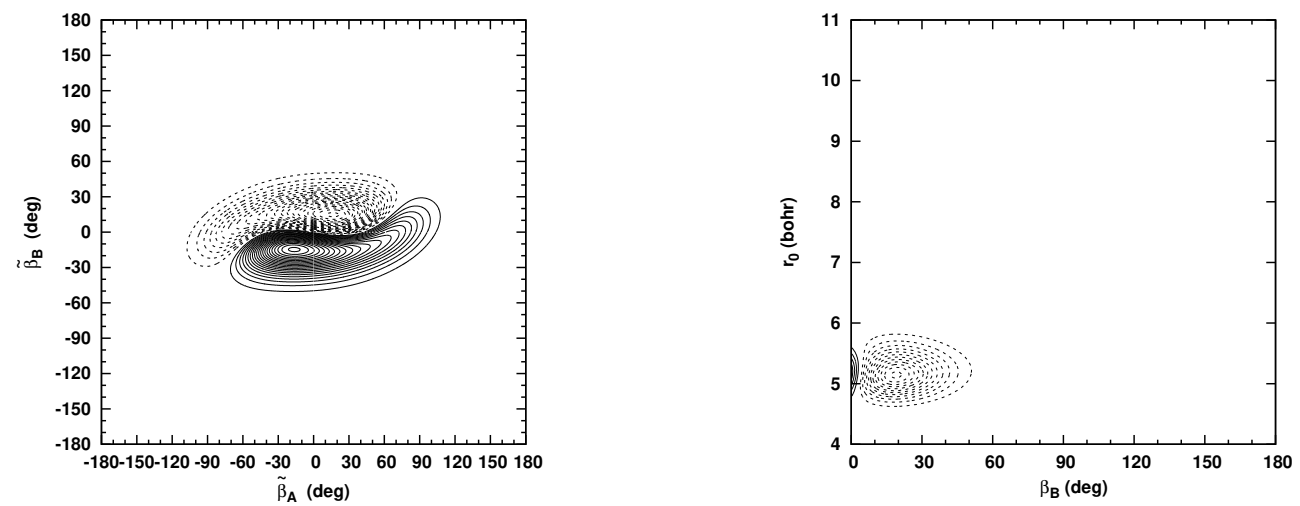

FIG. 8: Wavefunction cut plots for the $\nu_{B(o)}\left(B^{-}\right)$state at $577.164 \mathrm{~cm}^{-1}$.

Fig. 7 shows wavefunction plots for the $\nu_{\beta(i)}$ and $\nu_{B(i)}$ in-plane bend states. Both these fundamentals have $B^{+}$symmetry. The first is a bending mode in which atoms at the two ends of the hydrogen bond move in the same direction and the second is a bending mode in which the same two atoms move in opposite directions. The $2 \mathrm{D}$ plots show that $\left(\alpha_{A}-\alpha_{B}, \gamma_{A}\right)$ are coupled. The $\nu_{\beta(i)}$ state seems to correspond to motion that is slightly more along the $\alpha_{A}-\alpha_{B}$ coordinate; the $\nu_{B(i)}$ states seems to correspond to motion that is slightly more along the $\gamma_{A}$ coordinate. The $\nu_{B(o)}$ state in Fig. 8 is of $B^{-}$symmetry and has a clear node in $\beta_{B}$. Fig. 9 shows the first excited state with $A^{+}$symmetry. It has stretch character and is labelled $\nu_{\sigma}$. 
TABLE VI: $B^{+}$and $A^{-}$vibrational levels $\left(\right.$in $\mathrm{cm}^{-1}$ ) computed using the basis defined by $j_{1 x}=$ $j_{2 x}=15, n_{r_{0}}=120$, and $r_{0} \in[4,11]$ bohr. The vibrational levels are relative to the zero-point energy (ZPE) at $1080.166 \mathrm{~cm}^{-1}$.

\begin{tabular}{|c|c|c|c|}
\hline Assignment & $B^{+}$ & Assignment & $A^{-}$ \\
\hline$\nu_{\beta(i)}$ & 196.131 & $\nu_{\beta(o)}+\nu_{\beta(i)}$ & 299.073 \\
\hline$\nu_{\sigma}+\nu_{\beta(i)}$ & 389.844 & $\nu_{\beta(o)}+\nu_{\sigma}+\nu_{\beta(i)}$ & 495.276 \\
\hline $2 \nu_{\beta(o)}+\nu_{\beta(i)}$ & 459.312 & $3 \nu_{\beta(o)}+\nu_{\beta(i)}$ & 622.011 \\
\hline $3 \nu_{\beta(i)}$ & 566.501 & $\nu_{\beta(o)}+2 \nu_{\sigma}+\nu_{\beta(i)}$ & 680.180 \\
\hline $2 \nu_{\sigma}+\nu_{\beta(i)}$ & 574.142 & $\nu_{\beta(o)}+3 \nu_{\beta(i)}$ & 721.462 \\
\hline $2 \nu_{\beta(o)}+\nu_{\sigma}+\nu_{\beta(i)}$ & 652.936 & $\nu_{\beta(i)}+\nu_{B(o)}$ & 743.984 \\
\hline$\nu_{B(i)}$ & 679.791 & $\nu_{\beta(o)}+\nu_{B(i)}$ & 794.478 \\
\hline $3 \nu_{\sigma}+\nu_{\beta(i)}$ & 743.350 & $3 \nu_{\beta(o)}+\nu_{\beta(i)}+\nu_{\sigma}$ & 811.908 \\
\hline$\nu_{\sigma}+3 \nu_{\beta(i)}$ & 755.495 & $\nu_{\beta(o)}+3 \nu_{\sigma}+\nu_{\beta(i)}$ & 854.195 \\
\hline $4 \nu_{\beta(o)}+\nu_{\beta(i)}$ & 788.486 & $\nu_{\beta(o)}+\nu_{\sigma}+3 \nu_{\beta(i)}$ & 908.755 \\
\hline$\nu_{\sigma}+\nu_{B(i)}$ & 854.768 & $4 \nu_{\sigma}+\nu_{\beta(o)}+\nu_{\beta(i)}$ & 1018.061 \\
\hline $2 \nu_{\beta(o)}+3 \nu_{\beta(i)}$ & 882.465 & & \\
\hline $4 \nu_{\sigma}+\nu_{\beta(i)}$ & 905.960 & & \\
\hline $5 \nu_{\beta(i)}$ & 940.476 & & \\
\hline$\nu_{\sigma}+4 \nu_{\beta(o)}+\nu_{\beta(i)}$ & 973.043 & & \\
\hline \\
\hline$\nu_{\sigma}+2 \nu_{\beta(o)}+3 \nu_{\beta(i)}$ & 1054.489 & & \\
\hline $5 \nu_{\sigma}+\nu_{\beta(i)}$ & 1057.301 & & \\
\hline
\end{tabular}

\section{B. Computed rovibrational levels and their $J\left(K_{a}, K_{c}\right)$ assignments}

We computed ro-vibrational levels for $J$ values up to $J=4$ and the rotational transitions are reported in Tables 1 and 2 of the supplementary material. Both tables include comparisons with experimental values $[12,16]$ for ground, $\nu_{\beta o}, \nu_{\beta i}$ and $\nu_{\sigma}$ states. Many of our ro-vibrational levels can be assigned to particular vibrational states. The levels are la- 


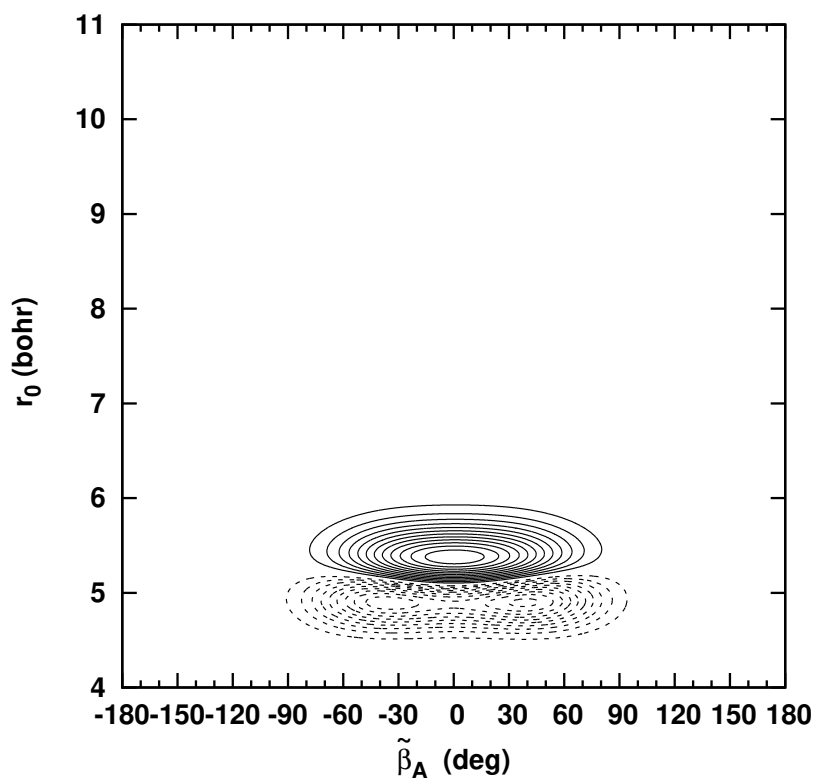

FIG. 9: Wavefunction cut $\left(\tilde{\beta_{A}}, r_{0}\right)$ for the $A^{+}$state at $195.998 \mathrm{~cm}^{-1}$.

belled by $K$, which is the quantum number for the projection of the angular momentum on the inter-monomer. Assigning $K$ labels is straightforward because our basis functions are labelled by $K . K=K_{a}$ in standard spectroscopic notation. $\mathrm{H}_{2} \mathrm{O}-\mathrm{HF}$ is a nearly prolate asymmetric rotor and we can therefore assign $K_{c}$ values by assuming the standard prolate energy order.

In the following three subsections, we focus attention on the three $J=2 \leftarrow 1$ transitions that occur within each of the vibrational states. These $R(1)$ transitions are $2_{12} \leftarrow 1_{11}, 2_{11} \leftarrow$ $1_{10}$ and $2_{02} \leftarrow 1_{01}$, forming $R(1)$ triplets in which, in the absence of perturbations, the $K=0$ line is in the middle. Kisiel et al. observe and assign $R(1)$ transitions to many vibrational states using Stark effect patterns, relative intensity measurements, and nuclear spin statistical weights. We organize the observed $R(1)$ triple lines into three groups (See Table IX). The first group consists of the $R(1)$ lines of the ground state and $\nu_{\beta o}$, and $\nu_{\beta i}$ states. The second group consists of the $R(1)$ lines of the $2 \nu_{\beta o}, 2 \nu_{\beta i}, \nu_{\beta o}+\nu_{\beta i}$ states. They are transitions in vibrational states with two bend excitations and are in the same region as those the first group, but about an order of magnitude weaker. In particular, we discuss a possible reassignment of Kisiel et al.'s satellite A and B bands in this region. The third group consists of the $R(1)$ lines of vibrational states involving $\nu_{\sigma}$. They are red-shifted from 
the region of the first and second groups. Wavefunction plots (for $J=0$ ) and computed intensities (for $J>0$ ) are used to make the vibrational assignments. We compare our $R(1)$ triplet assignments with the experimental assignments of Kisiel et al.. Tables 3 and 4 of the supplementary material give $J \leq 4$ rotational levels for different vibrational states. Tables VII and VIII give rotational transition frequencies for states up to $J=4$, with corresponding intensities, for the ground, $\nu_{\beta o}, \nu_{\beta i}, \nu_{\sigma}$ and $2 \nu_{\beta o}, 2 \nu_{\beta i}, 2 \nu_{\sigma}$ states.

TABLE VII: Computed intensities at $\mathrm{T}=230 \mathrm{~K}$ for rotational transitions up to $J=4$, in the ground, $\nu_{\beta o}, \nu_{\beta i}$. and $\nu_{\sigma}$ states. All rotational frequencies are given in $\mathrm{cm}^{-1}$ and intensities in $\left(\mathrm{e} \mathrm{a}_{0}\right)^{2}$.

\begin{tabular}{lll|ll|ll|lll}
\hline \multicolumn{3}{c}{ GS } & \multicolumn{3}{c}{$\nu_{\beta o}$} & \multicolumn{3}{c}{$\nu_{\beta i}$} & \multicolumn{2}{c}{$\nu_{\sigma}$} \\
Transition & Freq. $\left(\mathrm{cm}^{-1}\right)$ & Intensity & Freq. $\left(\mathrm{cm}^{-1}\right)$ & Intensity & Freq. $\left(\mathrm{cm}^{-1}\right)$ & Intensity & Freq. $\left(\mathrm{cm}^{-1}\right)$ & Intensity \\
\hline $1-01<0-00$ & 0.4723 & 0.0023 & 0.4761 & 0.0012 & 0.4738 & 0.0007 & 0.4610 & 0.0006 \\
$2-02<1-01$ & 0.9445 & 0.0182 & 0.9522 & 0.0277 & 0.9475 & 0.0155 & 0.9219 & 0.0050 \\
$2-12<1-11$ & 0.9402 & 0.0372 & 0.9488 & 0.0065 & 0.9419 & 0.0034 & 0.9187 & 0.0102 \\
$2-11<1-10$ & 0.9482 & 0.0378 & 0.9549 & 0.0065 & 0.9525 & 0.0035 & 0.9260 & 0.0104 \\
$3-03<2-02$ & 1.4167 & 0.0610 & 1.4282 & 0.0926 & 1.4212 & 0.0518 & 1.3827 & 0.0167 \\
$3-13<2-12$ & 1.4102 & 0.1477 & 1.4231 & 0.0256 & 1.4127 & 0.0136 & 1.3779 & 0.0405 \\
$3-12<2-11$ & 1.4222 & 0.1502 & 1.4323 & 0.0259 & 1.4286 & 0.0139 & 1.3889 & 0.0413 \\
$3-22<2-21$ & 1.4148 & 0.0238 & 1.4266 & 0.0396 & 1.4195 & 0.0187 & 1.3872 & 0.0065 \\
$3-21<2-20$ & 1.4148 & 0.0238 & 1.4266 & 0.0396 & 1.4195 & 0.0187 & 1.3872 & 0.0065 \\
$4-04<3-03$ & 1.8887 & 0.1431 & 1.9041 & 0.2178 & 1.8948 & 0.1216 & 1.8435 & 0.0392 \\
$4-14<3-13$ & 1.8801 & 0.3654 & 1.8973 & 0.0633 & 1.8835 & 0.0337 & 1.8370 & 0.1002 \\
$4-13<3-12$ & 1.8961 & 0.3717 & 1.9095 & 0.0641 & 1.9047 & 0.0344 & 1.8517 & 0.1018 \\
$4-23<3-22$ & 1.8863 & 0.0755 & 1.9020 & 0.1254 & 1.8926 & 0.0593 & 1.8494 & 0.0206 \\
$4-22<3-21$ & 1.8863 & 0.0755 & 1.9020 & 0.1254 & 1.8926 & 0.0593 & 1.8494 & 0.0206 \\
$4-32<3-31$ & 1.8832 & 0.0849 & 1.8986 & 0.0170 & 1.8875 & 0.0069 & 1.8725 & 0.0231 \\
$4-31<3-30$ & 1.8832 & 0.0849 & 1.8986 & 0.0170 & 1.8875 & 0.0069 & 1.8725 & 0.0231 \\
\hline
\end{tabular}

\section{Rotational transition frequencies in the ground state, $\nu_{\beta o}, \nu_{\beta i}$}

There are extensive experimental results for the ground, $\nu_{\beta o}$, and $\nu_{\beta i}$ states. The calculated (full lines) and observed (dashed lines) frequency positions for $J=2 \leftarrow 1$ transitions are given in Tab. IX and schematically illustrated in Fig. 10 by a stick spectrum computed using intensities at $\mathrm{T}=230 \mathrm{~K}$ and including nuclear statistical spin weights. For all these bands, we observe a strong $R(1)$ triplet. The $K=0$ transition is in the middle, as would be the case for a rigid prolate top. The line strengths for $K=1$ transitions are nearly equal.

The difference between theoretical and experimental frequencies comes from two sources. 
TABLE VIII: Computed intensities at $\mathrm{T}=230 \mathrm{~K}$ for rotational transitions up to $J=4$, in the $2 \nu_{\beta o}, 2 \nu_{\beta i}$ and $2 \nu_{\sigma}$ vibrational states. All rotational frequencies are given in $\mathrm{cm}^{-1}$ and intensities in $\left(\mathrm{e} \mathrm{a}_{0}\right)^{2}$.

\begin{tabular}{lll|ll|ll}
\hline & \multicolumn{3}{c}{$2 \nu_{\beta o}$} & \multicolumn{2}{c}{$2 \nu_{\beta i}$} \\
Transition & Freq. $\left(\mathrm{cm}^{-1}\right)$ & Intensity & Freq. $\left(\mathrm{cm}^{-1}\right)$ & Intensity & Freq. $\left(\mathrm{cm}^{-1}\right)$ & Intensity \\
\hline $1-01<0-00$ & 0.4741 & 0.0004 & 0.4683 & 0.0002 & 0.4548 & 0.0002 \\
$2-02<1-01$ & 0.9482 & 0.0033 & 0.9368 & 0.0015 & 0.9093 & 0.0015 \\
$2-12<1-11$ & 0.9447 & 0.0070 & 0.9433 & 0.0029 & 0.8944 & 0.0030 \\
$2-11<1-10$ & 0.9492 & 0.0070 & 0.9552 & 0.0029 & 0.9016 & 0.0030 \\
$3-03<2-02$ & 1.4223 & 0.0109 & 1.4057 & 0.0049 & 1.3633 & 0.0050 \\
$3-13<2-12$ & 1.4170 & 0.0276 & 1.4149 & 0.0114 & 1.3416 & 0.0119 \\
$3-12<2-11$ & 1.4237 & 0.0279 & 1.4328 & 0.0117 & 1.3525 & 0.0121 \\
$3-22<2-21$ & 1.4149 & 0.0050 & 1.4253 & 0.0016 & 1.3471 & 0.0019 \\
$3-21<2-20$ & 1.4149 & 0.0050 & 1.4253 & 0.0016 & 1.3471 & 0.0019 \\
$4-04<3-03$ & 1.8963 & 0.0256 & 1.8750 & 0.0116 & 1.8167 & 0.0117 \\
$4-14<3-13$ & 1.8893 & 0.0683 & 1.8864 & 0.0282 & 1.7886 & 0.0295 \\
$4-13<3-12$ & 1.8981 & 0.0689 & 1.9102 & 0.0289 & 1.9025 & 0.0248 \\
$4-23<3-22$ & 1.8864 & 0.0158 & 1.9000 & 0.0051 & 1.7959 & 0.0061 \\
$4-22<3-21$ & 1.8864 & 0.0158 & 1.9000 & 0.0051 & 1.7959 & 0.0061 \\
$4-32<3-31$ & 1.8592 & 0.0209 & 1.8097 & 0.0068 & 1.8964 & 0.0114 \\
$4-31<3-30$ & 1.8592 & 0.0209 & 1.8097 & 0.0068 & 1.8964 & 0.0114 \\
\hline
\end{tabular}

There is a rigid-rotor contribution; even if the molecule is rigid, theoretical and experimental frequencies will differ if the theoretical geometry is not perfect. There is a ro-vibrational contribution due to coupling between vibration and rotation. The rigid-rotor contribution is clearly the same for all vibrational states. In the absence of resonances, the similarity of the ro-vibrational contributions for two vibrational states will depend on the similarity of the vibrations. The ro-vibrational contributions for two vibrational states will be more different if one of the two states is more excited. The difference between theory and experiment for all three of the $R(1)$ transitions for the ground state is $0.016 \mathrm{~cm}^{-1}$ (see Obs-Calc in Tab. IX). The difference between theory and experiment for the three $R(1)$ transitions for $\nu_{\beta o}$ and $\nu_{\beta i}$ are also close to $0.016 \mathrm{~cm}^{-1}$ (see Obs-Calc in Tab. IX). This means that the relative position of the ground state, $\nu_{\beta o}, \nu_{\beta i}$ are accurately predicted by our calculation, giving us confidence that the relative position of the weaker vibrational bands would also be accurate. In Fig. 10, the computed frequencies for all three vibrational states are shifted by $0.016 \mathrm{~cm}^{-1}$.

Resonances might cause the theoretical and experimental frequencies to differ by an amount different from $0.016 \mathrm{~cm}^{-1}$. Belov et al. [16] fit the spectrum by including a Coriolis 


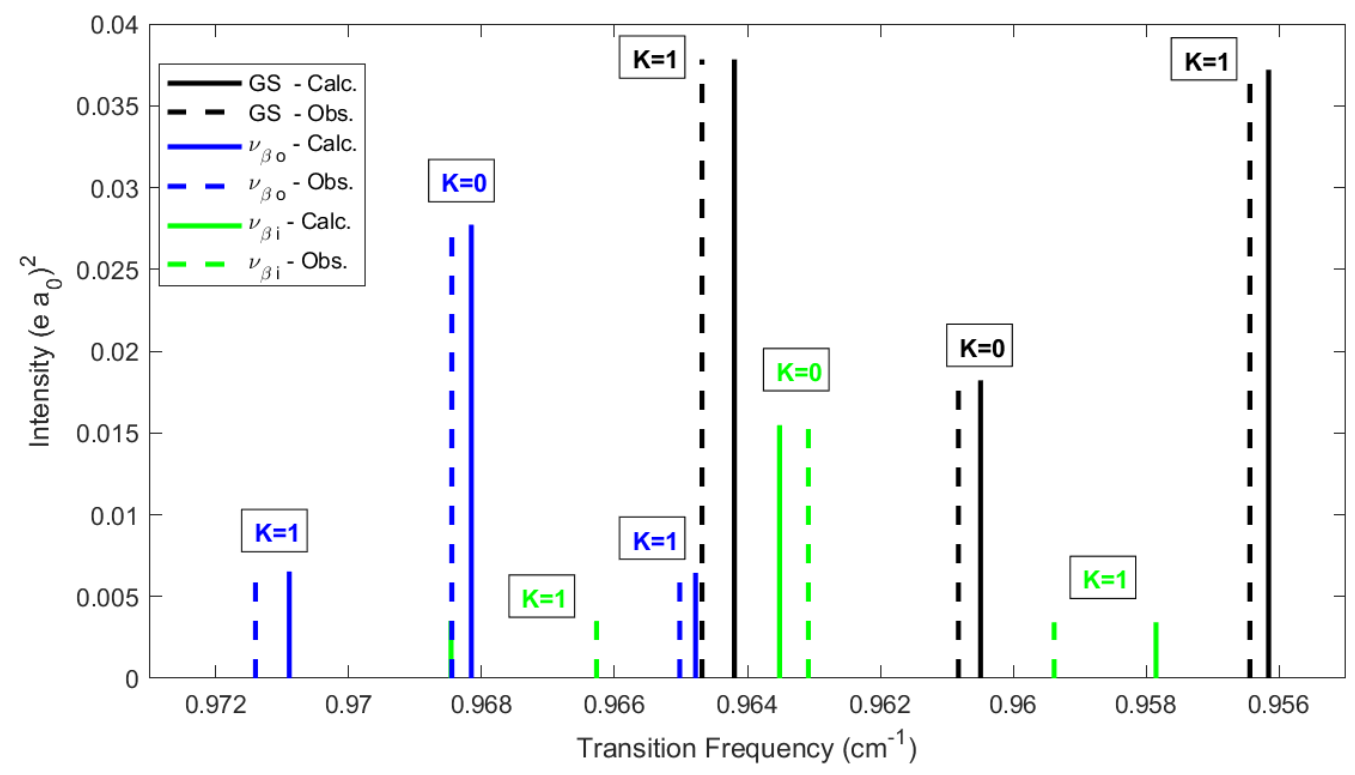

FIG. 10: $J=2 \leftarrow 1$ transitions of $\mathrm{H}_{2} \mathrm{O}-\mathrm{HF}$ in the ground, $\nu_{\beta o}$, and $\nu_{\beta(i)}$ states. All calculated positions are shifted by an average shift of $0.016 \mathrm{~cm}^{-1}$ calculated from $R(1)$ GS transitions.

interaction between the ground and $\nu_{\beta o}$ states and revealed the existence of an important resonance. As discussed by Cazzoli et al.[12] and later by Belov et al.[16], we observe that the $K_{a}=3$ level of the ground state (with energy of $125.905 \mathrm{~cm}^{-1}$ ) and the $K_{a}=2$ level of the $\nu_{\beta o}$ (with energy of $129.620 \mathrm{~cm}^{-1}$ ) for the rotational state $J=4$ have very similar energies. Belov et al. assign $4_{23} \leftarrow 3_{22}$ and $4_{22} \leftarrow 3_{21}$ to transitions at 57.9822 and $57.9322 \mathrm{GHz}$ for $\nu_{\beta o}$, but this is probably a misprint, both should be $57.9822 \mathrm{GHz}$. Cazzoli et al. assigned both $4_{23} \leftarrow 3_{22}$ and $4_{22} \leftarrow 3_{21}$ to transitions at $58.0317 \mathrm{GHz}$. Our calculations slightly favor Belov et al's assignment (see Obs.-Calc. of Table 1 of the supplementary material).

D. Assignment and $R(1)$ rotational transition frequencies in the $2 \nu_{\beta o}, 2 \nu_{\beta i}, \nu_{\beta o}+\nu_{\beta i}$, and $3 \nu_{\beta o}$ states

Much weaker $R(1)$ transitions, in the same region as those in Figure 10, are shown in Figure 11. Kisiel et al. assign their A and B satellites to $2 \nu_{\beta o}$ and $\nu_{\beta o}+\nu_{\beta i}$, respectively. $[7,8]$ Their $2 \nu_{\beta o} R(1)$ frequencies are higher than their $\nu_{\beta o}+\nu_{\beta i} R(1)$ frequencies. In our calculated spectrum the order is reversed. On the other hand, the order of the calculated and observed 
$\nu_{\beta o}$ and $\nu_{\beta i}$ lines is the same. This suggests that the PES and the calculate are accurate and that Kisiel et al.'s assignment might be wrong. We suggest that it is possible that Kisiel et al.'s assignment of the A and B satellites might be interchanged: satellite A could be $\nu_{\beta o}+\nu_{\beta i}$ and satellite $\mathrm{B}$ could be $2 \nu_{\beta o}$. Interchanging the experimental assignments also improves the agreement between the experimental and our calculated vibrational energies. After exchanging the assignments the experimental vibrational energies of $\nu_{\beta o}+\nu_{\beta i}$ and $2 \nu_{\beta o}$ become $267 \pm 35$ and $228 \pm 15 \mathrm{~cm}^{-1}$. Our calculated values are 299.1 and $238.2 \mathrm{~cm}^{-1}$.

Kisiel et al. prefer their assignment of satellites A and B for three reasons. The first reason is based on observed dipole moments. Kisiel et al. observe that the dipole moments of $\nu_{\beta i}$ and the ground state are very similar, indicating that exciting $\nu_{\beta i}$ has little effect on the dipole moment. On the basis of this observation, they expect the dipole moment of $\nu_{\beta o}+\nu_{\beta i}$ to be close to the dipole moment of $\nu_{\beta o}$. However, because $\nu_{\beta o}$ is the tunnelling mode, it is risky to assume that because the dipole moment of $\nu_{\beta o}$ is significantly less than the dipole moment of the ground state that the dipole moment of $2 \nu_{\beta o}$ will be significantly less than the dipole moment of $\nu_{\beta o}$. The second reason is based on the idea that for the satellite that is $2 \nu_{\beta o}$ a ratio of differences of rotational constants should be similar for the $B$ and $C$ rotational constants. In fact, ratios calculated from the $B$ and $C$ rotational constants we compute (see section VI F and Table IX ) are similar for neither $\nu_{\beta o}+\nu_{\beta i}$ nor $2 \nu_{\beta o}$. Kisiel et al.'s third reason is that if they assume that Satellite A is $2 \nu_{\beta o}$, they find that their observed ratio of rotational constant differences is consistent with the 1-D double well potential that they extract from their $\nu_{\beta o}$ transition frequencies. This 1-D model might not be reliable.

Also in figure 11, for $2 \nu_{\beta i}$ the $K=0$ transition is not between the two $K=1$ transitions, as it would be for a rigid rotor; this is a sure sign that perturbations are important. According to our calculations, (grey lines in Fig. 11) there are strong $K=0$ transitions in the $\nu_{\beta o}+2 \nu_{\beta i}, 2 \nu_{\beta o}+\nu_{\beta i}$ and $3 \nu_{\beta i}$ states in same spectral region. There are no corresponding experimental values. Kisiel et al. assigned a weak line they called satellite C (p.164 of Kisiel's thesis [17]) to a $B$ state with $K=0$ and three quanta. We also tentatively assign it as $3 \nu_{\beta o}$. The $3 \nu_{\beta i} K=0$ is close, but it is a $A$ symmetry line. 


\section{E. $\quad R(1)$ transition frequencies for vibrational states involving $\nu_{\sigma}$}

Rotational transitions within all vibrational states with a contribution from $\nu_{\sigma}$ are redshifted with respect to the ground state transition frequencies (see Fig. 12). In our calculated spectrum, we have assigned $R(1)$ triplets for $\nu_{\sigma}, \nu_{\beta o}+\nu_{\sigma}, \nu_{\beta i}+\nu_{\sigma}, 2 \nu_{\sigma}, \nu_{\sigma}+2 \nu_{\beta o}$ and $2 \nu_{\sigma}+2 \nu_{\beta o}$ states. The experimental positions of the $3 R(1)$ lines are known only for $\nu_{\sigma}$ and for other vibrational states some of the $R(1)$ lines were not observed. The shifted spectrum agrees well with the available experimental rotational transitions. In our calculation, the $K=0$ $\nu_{\beta i}+\nu_{\sigma}$ transition frequency is smaller than the two $K=1$ frequencies. This is an indication of important perturbations. Kisiel suggests that the $\nu_{\sigma} R(1)$ triplet is perturbed due to possible anharmonic vibrations. We confirm that the $K=0$ transition is not exactly in the middle of the two $K=1$ transitions, which again indicates a perturbation.

\section{F. $\quad A, B, C$ rotational constants for vibrational states derived from $J=1$ levels}

For each vibrational state, we calculate rotational constants from the three $J=1$ levels assuming the $J=1$ levels are solely determined by the rotational constants. The rotational constants encode information about the rotational levels in a compact form. The derived rotational constants are given in column 2 of Table IX. Unlike rotational constants $B$ and $C$, there are no experimental data for rotational constant $A$. We find that the variation of the $A$ rotational constant with respect to vibration is large. We find that, compared to the ground state, $A$ decreases for $\nu_{\beta}(o), A$ increases for $\nu_{\beta}(i), A$ does not change much for $\nu_{\sigma}$. The variations can be qualitative explained. Rotational constant $A$ depends on how far the hydrogen atoms are from the $a$-axis (approximately aligned with the inter-monomer axis). For $\nu_{\beta}(o)$, the ground state wavefunction (see Fig. 5) peaks at the saddle point of the double well and the $\nu_{\beta}(o)$ vibration takes all three $\mathrm{H}$ atoms away from the $a$-axis and hence causes $A$ to decrease. Since $\nu_{\sigma}$ is a stretch vibration, it does change $A$ by much. For $\nu_{\beta}(i)$, it is harder to explain the increased $A$ constant. From the wavefunction plot (Figure 7 ) it is apparent that the $\nu_{\beta}(i)$ vibration involves changing $\gamma_{A}$. It is somewhat similar to the acceptor twist vibration of $\mathrm{H}_{2} \mathrm{O}$ dimer which also an increases the $A$ constant[59]. The $\nu_{\beta}(i)$ vibration also involves changes in $\beta_{A}$ and $\alpha_{A}-\alpha_{B}$ (the latter is shown in Fig. 7). Changing them from their saddle point values decreases the distance of one $\mathrm{H}$ atom of $\mathrm{H}_{2} \mathrm{O}$ from the $a$-axis but 
increases the distance of the other $\mathrm{H}$ atom. On average, it must move the $\mathrm{H}$ atoms closer to the axis.

\section{G. Vibrational energies}

There are large differences between our calculated vibrational energies and those of Kisiel et al.[7] and Legon et al.[9]. The experimental vibrational energies are obtained from relative intensity measurements and may have significant error. Kisiel et al. 's $2 \nu_{\beta(o)}$ vibrational state is at $267 \pm 35 \mathrm{~cm}^{-1}$, and their $\nu_{\beta o}+\nu_{\beta i}$ at $228 \pm 15 \mathrm{~cm}^{-1}$. Our calculated energies are 238.2 $\mathrm{cm}^{-1}$ for $2 \nu_{\beta_{o}}$ and $299.1 \mathrm{~cm}^{-1}$ for $\nu_{\beta o}+\nu_{\beta i}$. However in section VI D, we suggest interchanging the experimental assignments of these bands. With this interchange, the order of these two bands is the same in both theory and experiment. For the $2 \nu_{\beta_{i}}$ vibrational state, Legon et al. [9] give the energy of $330 \pm 30 \mathrm{~cm}^{-1}$ which should be compared with our theoretical value of $386.0 \mathrm{~cm}^{-1}$. Clearly, the differences for the $2 \nu_{\beta_{o}}, 2 \nu_{\beta_{i}}$ and $\nu_{\beta(o)}+\nu_{\beta(i)}$ states are large.

For the two highest bending fundamentals $\nu_{B(o)}=666 \pm 30 \mathrm{~cm}^{-1}$ and $\nu_{B(i)}=696 \pm 30$ $\mathrm{cm}^{-1}$ determined by Thomas et al.[3], our calculated values are 577.2 and $679.8 \mathrm{~cm}^{-1}$. Our calculated $\nu_{B(o)}$ frequency is not within the uncertainty limits of the experimental result, but our calculated $\nu_{B(i)}$ frequency is in good agreement with the experimental result.

\section{H. Comparison with Loreau's 5D PES [20]}

While this study was being completed, Loreau et al. published a 5-D PES and calculated some ro-vibrational levels. [20] We have done calculations on their 5-D PES. Loreau et al. use standard polyspherical coordinates. For $\mathrm{H}_{2} \mathrm{O}$, we use the same monomer geometry (averaged over the ground-state vibrational wavefunction) as Loreau et al., but for $r_{H F}$ our bond length is different. Loreau used $\left\langle r^{-2}\right\rangle^{-1 / 2}=1.74952 \mathrm{bohr}$, derived from the experimental rotational constant [60] whereas our $r_{H F}$ is $\langle r\rangle=1.76286$ bohr (see Table IV of Ref. [45] ). Our choice is consistent because we use $\langle r\rangle$ for both $\mathrm{HF}$ and $\mathrm{H}_{2} \mathrm{O}$. In Table $\mathrm{X}$, we compare the published bound states from Table I of Ref. [20] (with an estimated convergence error of $0.05 \mathrm{~cm}^{-1}$ ) with our energies on the same PES and the energies on the PES of this paper. The two PESs are evidently very similar. The vibrational band origin of the $\nu_{\beta o}$ band is $78.9 \mathrm{~cm}^{-1}$ on the Loreau et al. PES and $81.9 \mathrm{~cm}^{-1}$ on the PES of this paper. 
The only experimental determination of this band origin was made by Kisiel et al. To obtain a band origin, Loreau et al. subtract the energy of the lowest ortho state assigned to the ground vibrational state $\left(14.00 \mathrm{~cm}^{-1}\right)$ from their lowest $\nu_{\beta o}$ energy $\left(78.92 \mathrm{~cm}^{-1}\right)$. However, Kisiel et al.'s band origin of $64 \pm 10 \mathrm{~cm}^{-1}$ is derived from the relative intensity of two rotational transitions in the ground state and in $\nu_{\beta o}$. Although this procedure involves approximations, it yields the energy of $\nu_{\beta o}$ relative to the energy of the ground vibrational state. To compare with experiment, one should not subtract the energy of the lowest ortho state. Both our vibrational band origin and that of Loreau et al. are outside the range determined by the error bars of the experimental value. It is clearly necessary to directly measure the vibrational energy of this important tunnelling state via direct far-infrared spectroscopy in order to determine whether the PES of this work or the PES of Loreau et al. is more accurate, and whether a full dimensional (9D) calculation is required.

\section{CONCLUSION}

In this paper we use a new PES to compute the rovibrational spectrum of $\mathrm{H}_{2} \mathrm{O}-\mathrm{HF}$. The new PES is a 9-D surface, but we use the rigid monomer approximation to obtain the spectrum. $\mathrm{H}_{2} \mathrm{O}-\mathrm{HF}$ has two equivalent minima separated by a low barrier. It is therefore important to use basis functions that have significant amplitude in large regions of the configuration space of the molecule. Intensities help us to assign states we compute. To compare with experimental results we focus on the $R(1)$ transitions in various vibrational states. The agreement between theory and experiments is good and the difference between theory and experiment is nearly the same for many vibrational states. This allows us to confirm some of the assignments made by experimentalists and suggest a possible reassignment for two bands. Agreement between experimental and theoretical vibrational energies is less good. Most of the experimental vibrational energies are obtained from relative intensity measurements and are rather imprecise. $\mathrm{H}_{2} \mathrm{O}-\mathrm{HF}$ is an excellent candidate for more experimental high-resolution studies. 9-D calculations are possible. 


\section{Acknowledgments}

This work has been supported by the Natural Sciences and Engineering Research Council of Canada. Calculations were done on a computer purchased from a Canadian Foundation for Innovation grant and on a SciNet computer (Compute Canada) at University of Toronto.

[1] W. Klemperer and V. Vaida, Proceedings of the National Academy of Sciences of the United States of America 103, 10584 (2006).

[2] J. Bevan, A. Legon, D. Millen, and S. Rogers, Journal of the Chemical Society, Chemical Communications pp. 341-343 (1975).

[3] R. K. Thomas, Proceedings of the Royal Society of London. Series A, Mathematical and PhysicalSciences 344, 579 (1975).

[4] D. Lister and P. Palmiari, J. Mol. Struct. 39, 295 (1977).

[5] J. Bevan, Z. Kisiel, A. Legon, D. Millen, and S. Rogers, Proc. R. Soc. Lond. A pp. 441-451 (1980).

[6] Z. Kisiel, A. Legon, and D. Millen, J. Mol. Struct. 112, 1 (1984).

[7] Z. Kisiel, A. Legon, and D. Millen, Proc.R.Soc.Lond. A 381, 419 (1982).

[8] Z. Kisiel, A. Legon, and D. Millen, J. Chem. Phys. 78, 2910 (1983).

[9] A. Legon and D. Millen, Faraday Discuss. Chem. Soc. 73, 71 (1982).

[10] A. Legon and L. Willoughby, Chem. Phys. Lett. 92, 333 (1982).

[11] M. Szczesniak, S. Scheiner, and Y. Bouteiller, J. Chem. Phys. 81, 5024 (1984).

[12] G. Cazzoli, P. Favero, D. Lister, A. Legon, D. Millen, and Z. Kisiel, Chem. Phys. Lett. 117, $543(1985)$.

[13] A. Legon, D. Millen, and H. North, Chem. Phys. Lett. 135, 303 (1987).

[14] T. Sexton, J. Howard, and G. Tschumper, J. Phys. Chem. A 122, 4902 (2018).

[15] J. Demaison and J. Lievin, Mol. Phys. 106, 1249 (2008).

[16] S. Belov, V. Demkin, N. Zobov, E. Karyakin, A. Krupnov, I. Kozin, O. Polyansky, and M. Tretyakov, J. Mol. Spectrosc. 241, 124 (2007).

[17] Z. Kisiel, THESIS - An investigation of the microwave spectra 
of $\mathrm{R}_{2} \mathrm{O} \quad \ldots \quad \mathrm{HX}$ type hydrogen bonded dimers in the gas phase, http://info.ifpan.edu.pl/ kisiel/on23/1980_Kisiel_reduced.pdf (1980).

[18] X.-G. Wang and J. Carrington, T., J. Chem. Phys. 143 (2015).

[19] X.-G. Wang and T. Carrington Jr., J. Chem. Phys. 134 (2011).

[20] J. Loreau, Y. Kalugina, A. Faure, A. Van Der Avoird, and F. Lique, J. Chem. Phys. 153 (2020).

[21] A. Van Der Avoird and D. Nesbitt, J. Chem. Phys. 134 (2011).

[22] G. Brocks, A. V. Avoird, B. Sutcliffe, and J. Tennyson, Mol. Phys. 50, 1025 (1983).

[23] N. R. Zare, Angular Momentum: Understanding Spatial Aspects in Chemistry and Physics (Wiley, 1991).

[24] J. C. Light and T. Carrington Jr., Adv. Chem. Phys. 114, 263 (2000).

[25] J. Light, I. Hamilton, and J. Lill, J. Chem. Phys. 82, 1400 (1985).

[26] Z. Bacic and J. Light, Annu. Rev. Phys. Chem. 40 (1989).

[27] X.-G. Wang and J. Carrington, T., J. Chem. Phys. 146 (2017).

[28] A. van der Avoird, P. Wormer, and R. Moszynski, Chem. Rev. 94, 1931 (1994).

[29] P. Wormer and A. Van Der Avoird, Chem. Rev. 100, 4109 (2000).

[30] K. Raghavachari, G. W. Trucks, J. A. Pople, and M. Head-Gordon, Chem. Phys. Letters 157, 479 (1989).

[31] C. Hättig, D. P. Tew, and A. Köhn, J. Chem. Phys. 132, 231102 (2010).

[32] A. Köhn and D. P. Tew, J. Chem. Phys. 133, 174117 (2010).

[33] T. H. Dunning, Jr., J. Comp. Phys. 90, 1007 (1989).

[34] R. A. Kendall, T. H. Dunning, Jr., and R. J. Harrison, J. Chem. Phys. 96, 6796 (1992).

[35] D. P. Tew and W. Klopper, J. Chem. Phys. 123, 074101 (2005).

[36] F. A. Bischoff, S. Wolfsegger, D. P. Tew, and W. Klopper, Mol. Phys. 107, 963 (2009).

[37] T. A. Ruden, T. Helgaker, P. Jørgensen, and J. Olsen, J. Chem. Phys. 121, 5874 (2004).

[38] W. Mizukami, S. Habershon, and D. P. Tew, J. Chem. Phys. 141, 144310 (2014).

[39] W. Mizukami and D. P. Tew, J. Chem. Phys. 139, 194108 (2013).

[40] D. P. Tew and W. Mizukami, J. Phys. Chem. A 120, 9815 (2016).

[41] S. Erfort, M. Tschöpe, G. Rauhut, X. Zeng, and D. P. Tew, J. Chem. Phys. 152, 174306 (2020). 
[42] R. Tibshirani, J. Royal Statist. Soc. B 58, 267 (1996).

[43] G. Czako, E. Mátyus, and A. Császár, J. Phys. Chem. A. 113, 11665 (2009).

[44] M. Metz, K. Szalewicz, J. Sarka, R. Tóbiás, A. Császár, and E. Mátyus, Phys. Chem. Chem. Phys. 21, 13504 (2019).

[45] M. Jeziorska, P. Jankowski, K. Szalewicz, and B. Jeziorski, J. Chem. Phys. 113, 2957 (2000).

[46] T. Dyke, J. Chem. Phys. 66, 492 (1977).

[47] L. Braly, J. Cruzan, K. Liu, R. Fellers, and R. Saykally, J. Chem. Phys. 112, 10293 (2000).

[48] T. Carrington and X.-G. Wang, Wiley Interdisciplinary Reviews: Computational Molecular Science 1, 952 (2011).

[49] R. Dawes, X. G. Wang, and T. Carrington, J. Phys. Chem. A 117, 7612 (2013).

[50] P. Sarkar, N. Poulin, and T. Carrington Jr., J. Chem. Phys. 110, 10269 (1999).

[51] R. Toth, J. Opt. Soc. of Am. B 8, 2236 (1991).

[52] D. Webb and K. Narahari Rao, J. Mol. Spectrosc. 28, 121 (1968).

[53] I. Mills, T. Cvitas, H. K., K. N., and K.Kuchitsu, Quantities, Units and Symbols in Physical Chemistry (IUPAC, Blackwell, Oxford, 1993).

[54] X. G. Wang and T. Carrington, "Nonlinear-Nonlinear-Rigid monomers code for computing ro-vibrational spectra of Van der Waals molecules" (-).

[55] P. Jensen and P. R. Bunker, Molecular Symmetry and Spectroscopy (NRC Pres, 1998).

[56] C. Ruth Le Sueur, S. Miller, J. Tennyson, and B. Sutcliffe, Mol. Phys. 76, 1147 (1992).

[57] M. Smit, G. Groenenboom, P. Wormer, A. Van Der Avoird, R. Bukowski, and K. Szalewicz, J. Phys. Chem. A 105, 6212 (2001).

[58] P. Piecuch, V. Spirko, A. Kondo, and J. Paldusk, Mol. Phys. 94, 55 (1998).

[59] G. Groenenboom, P. Wormer, A. Van Der Avoird, E. Mas, R. Bukowski, and K. Szalewicz, J. Chem. Phys. 113, 6702 (2000).

[60] A. Mason and A. Nielsen, J. Opt. Soc.Am. 57, 1464 (1967). 
TABLE IX: $R(1)$ transitions in different vibrational states. The $\Delta^{1}$ column contains Obs.- Calc. values calculated using the experimental values and experimental assignments from Refs. [7, 12] for $G S, \nu_{\beta o}, \nu_{\beta i}, 2 \nu_{\beta i}$, and $\nu_{\sigma}$ and from Kisiel's thesis [17] for the other bands The $\Delta^{2}$ column contains Obs.- Calc.values calculated using the same experimental values but the the new possible assignment of this paper. The only entries in the $\Delta^{2}$ column are those for which the new assignment differs. $A, B, C$ rotational constants in the second column are calculated for each vibrational state in $\mathrm{cm}^{-1}$ from the three $J=1$ levels.

\begin{tabular}{|c|c|c|c|c|c|c|c|c|}
\hline Vib.state & $\begin{array}{l}A, B, C \\
\left(\mathrm{~cm}^{-1}\right)\end{array}$ & Transition & Calc. $\left(\mathrm{cm}^{-1}\right)$ & Obs. $\left(\mathrm{cm}^{-1}\right)$ & Obs.(MHz) & $\Delta^{1}$ & $\Delta^{2}$ & Intensity $\left(\mathrm{e} \mathrm{a}_{0}\right)^{2}$ \\
\hline GS & $\mathrm{A}=13.8221$ & $2-02 A^{+}<1-01 \mathrm{~A}^{-}$ & 0.9445 & $0.9608 \pm 0.000005$ & $28804.87 \pm 0.15$ & 0.016 & & 0.0182 \\
\hline GS & $\mathrm{B}=0.2381$ & $2-12 B^{+}<1-11 \mathrm{~B}^{-}$ & 0.9402 & $0.9564 \pm 0.000005$ & $28673.42 \pm 0.15$ & 0.016 & & 0.0372 \\
\hline GS & $\mathrm{C}=0.2341$ & $2-11 B^{-}<1-10 \mathrm{~B}^{+}$ & 0.9482 & $0.9647 \pm 0.000005$ & $28920.45 \pm 0.15$ & 0.016 & & 0.0378 \\
\hline$\nu_{\beta o}$ & $A=10.8227$ & $2-02 B^{-}<1-01 \mathrm{~B}^{+}$ & 0.9522 & $0.9684 \pm 0.00001$ & $29033.28 \pm 0.3$ & 0.016 & & 0.0277 \\
\hline$\nu_{\beta o}$ & $B=0.2396$ & $2-12 A^{-}<1-11 \mathrm{~A}^{+}$ & 0.9488 & $0.9650 \pm 0.00002$ & $28930.5 \pm 0.5$ & 0.016 & & 0.0064 \\
\hline$\nu_{\beta o}$ & $C=0.2365$ & $2-11 A^{+}<1-10 \mathrm{~A}^{-}$ & 0.9549 & $0.9714 \pm 0.00002$ & $29121.8 \pm 0.5$ & 0.016 & & 0.0065 \\
\hline$\nu_{\beta i}$ & $A=17.0142$ & $2-02 B^{+}<1-01 \mathrm{~B}^{-}$ & 0.9475 & $0.9631 \pm 0.00001$ & $28872.5 \pm 0.3$ & 0.016 & & 0.0155 \\
\hline$\nu_{\beta i}$ & $B=0.2395$ & $2-12 A^{+}<1-11 \mathrm{~A}^{-}$ & 0.9419 & $0.9594 \pm 0.00002$ & $28761.7 \pm 0.5$ & 0.018 & & 0.0034 \\
\hline$\nu_{\beta i}$ & $C=0.2342$ & $2-11 A^{-}<1-10 \mathrm{~A}^{+}$ & 0.9525 & $0.9663 \pm 0.00005$ & $28968.0 \pm 1.5$ & 0.014 & & 0.0035 \\
\hline $2 \nu_{\beta i}$ & $A=21.7161$ & $2-02 A^{+}<1-01 \mathrm{~A}^{-}$ & 0.9368 & & & & & 0.0015 \\
\hline $2 \nu_{\beta i}$ & $B=0.2372$ & $2-12 B^{+}<1-11 \mathrm{~B}^{-}$ & 0.9433 & $0.9631 \pm 0.0001$ & $28872.0 \pm 3.0$ & 0.020 & & 0.0029 \\
\hline $2 \nu_{\beta i}$ & $C=0.2312$ & $2-11 B^{-}<1-10 \mathrm{~B}^{+}$ & 0.9552 & $0.9682 \pm 0.00003$ & $29026.5 \pm 1.0$ & 0.013 & & 0.0029 \\
\hline $2 \nu_{\beta o}$ & $A=8.7511$ & $2-02 A^{+}<1-01 \mathrm{~A}^{-}$ & 0.9482 & & & & & 0.0033 \\
\hline $2 \nu_{\beta o}$ & $B=0.2382$ & $2-12 B^{+}<1-11 \mathrm{~B}^{-}$ & 0.9447 & $0.9667 \pm 0.00002$ & $28981.0 \pm 0.5$ & 0.022 & 0.010 & 0.0070 \\
\hline $2 \nu_{\beta o}$ & $C=0.2360$ & $2-11 B^{-}<1-10 \mathrm{~B}^{+}$ & 0.9492 & $0.9728 \pm 0.00002$ & $29164.0 \pm 0.5$ & 0.024 & 0.010 & 0.0070 \\
\hline$\nu_{\beta o}+\nu_{\beta i}$ & $A=12.0502$ & $2-02 A^{-}<1-01 \mathrm{~A}^{+}$ & 0.9551 & & & & & 0.0023 \\
\hline$\nu_{\beta o}+\nu_{\beta i}$ & $B=0.2401$ & $2-12 B^{-}<1-11 \mathrm{~B}^{+}$ & 0.9510 & $0.9552 \pm 0.00002$ & $28637.4 \pm 0.5$ & 0.004 & 0.016 & 0.0048 \\
\hline$\nu_{\beta o}+\nu_{\beta i}$ & $C=0.2375$ & $2-11 B^{+}<1-10 \mathrm{~B}^{-}$ & 0.9561 & $0.9604 \pm 0.00002$ & $28791.1 \pm 0.5$ & 0.004 & 0.017 & 0.0048 \\
\hline $3 \nu_{\beta o}$ & $A=12.4563$ & $2-02 B^{-}<1-01 \mathrm{~B}^{+}$ & 0.9516 & $0.9601 \pm 0.00002$ & $28781.8 \pm 0.5$ & 0.008 & & 0.0032 \\
\hline $3 \nu_{\beta o}$ & $B=0.2401$ & $2-12 A^{-}<1-11 \mathrm{~A}^{+}$ & 0.9278 & & & & & 0.0010 \\
\hline $3 \nu_{\beta o}$ & $C=0.2357$ & $2-11 A^{+}<1-10 \mathrm{~A}^{-}$ & 0.9191 & & & & & 0.0009 \\
\hline$\nu_{\sigma}$ & $A=13.5290$ & $2-02 A^{+}<1-01 \mathrm{~A}^{-}$ & 0.9219 & $0.9424 \pm 0.00002$ & $28253.7 \pm 0.5$ & 0.021 & & 0.0050 \\
\hline$\nu_{\sigma}$ & $B=0.2323$ & $2-12 B^{+}<1-11 \mathrm{~B}^{-}$ & 0.9187 & $0.9399 \pm 0.00002$ & $28179.2 \pm 0.5$ & 0.021 & & 0.0102 \\
\hline$\nu_{\sigma}$ & $C=0.2287$ & $2-11 B^{-}<1-10 \mathrm{~B}^{+}$ & 0.9260 & $0.9472 \pm 0.00002$ & $28397.2 \pm 0.5$ & 0.021 & & 0.0104 \\
\hline $2 \nu_{\sigma}$ & $A=14.3358$ & $2-02 A^{+}<1-01 \mathrm{~A}^{-}$ & 0.9093 & & & & & 0.0015 \\
\hline $2 \nu_{\sigma}$ & $B=0.2292$ & $2-12 B^{+}<1-11 \mathrm{~B}^{-}$ & 0.8944 & $0.9144 \pm 0.0001$ & $27414.3 \pm 3.0$ & 0.020 & & 0.0030 \\
\hline $2 \nu_{\sigma}$ & $C=0.2256$ & $2-11 B^{-}<1-10 \mathrm{~B}^{+}$ & 0.9016 & $0.9208 \pm 0.0001$ & $27605.7 \pm 3.0$ & 0.019 & & 0.0030 \\
\hline$\nu_{\sigma}+\nu_{\beta o}$ & $A=10.7342$ & $2-02 B^{-}<1-01 \mathrm{~B}^{+}$ & 0.9267 & $0.9434 \pm 0.00002$ & $28281.8 \pm 0.5$ & 0.017 & & 0.0075 \\
\hline$\nu_{\sigma}+\nu_{\beta o}$ & $B=0.2332$ & $2-12 A^{-}<1-11 \mathrm{~A}^{+}$ & 0.9231 & & & & & 0.0017 \\
\hline$\nu_{\sigma}+\nu_{\beta o}$ & $C=0.2301$ & $2-11 A^{+}<1-10 \mathrm{~A}^{-}$ & 0.9293 & & & & & 0.0018 \\
\hline$\nu_{\sigma}+\nu_{\beta i}$ & $A=12.0551$ & $2-02 B^{+}<1-01 \mathrm{~B}^{-}$ & 0.9213 & $0.9383 \pm 0.00002$ & $28130.2 \pm 0.5$ & 0.017 & & 0.0043 \\
\hline$\nu_{\sigma}+\nu_{\beta i}$ & $B=0.2310$ & $2-12 A^{+}<1-11 \mathrm{~A}^{-}$ & 0.9522 & & & & & 0.0008 \\
\hline$\nu_{\sigma}+\nu_{\beta i}$ & $C=0.2297$ & $2-11 A^{-}<1-10 \mathrm{~A}^{+}$ & 0.949730 & & & & & 0.0008 \\
\hline$\nu_{\sigma}+2 \nu_{\beta o}$ & $A=8.6074$ & $2-02 A^{+}<1-01 \mathrm{~A}^{-}$ & 0.9218 & & & & & 0.0009 \\
\hline$\nu_{\sigma}+2 \nu_{\beta o}$ & $B=0.2315$ & $2-12 B^{+}<1-11 \mathrm{~B}^{-}$ & 0.9184 & & & & & 0.0019 \\
\hline$\nu_{\sigma}+2 \nu_{\beta o}$ & $C=0.2294$ & $2-11 B^{-}<1-10 \mathrm{~B}^{+}$ & 0.9226 & & & & & 0.0019 \\
\hline
\end{tabular}



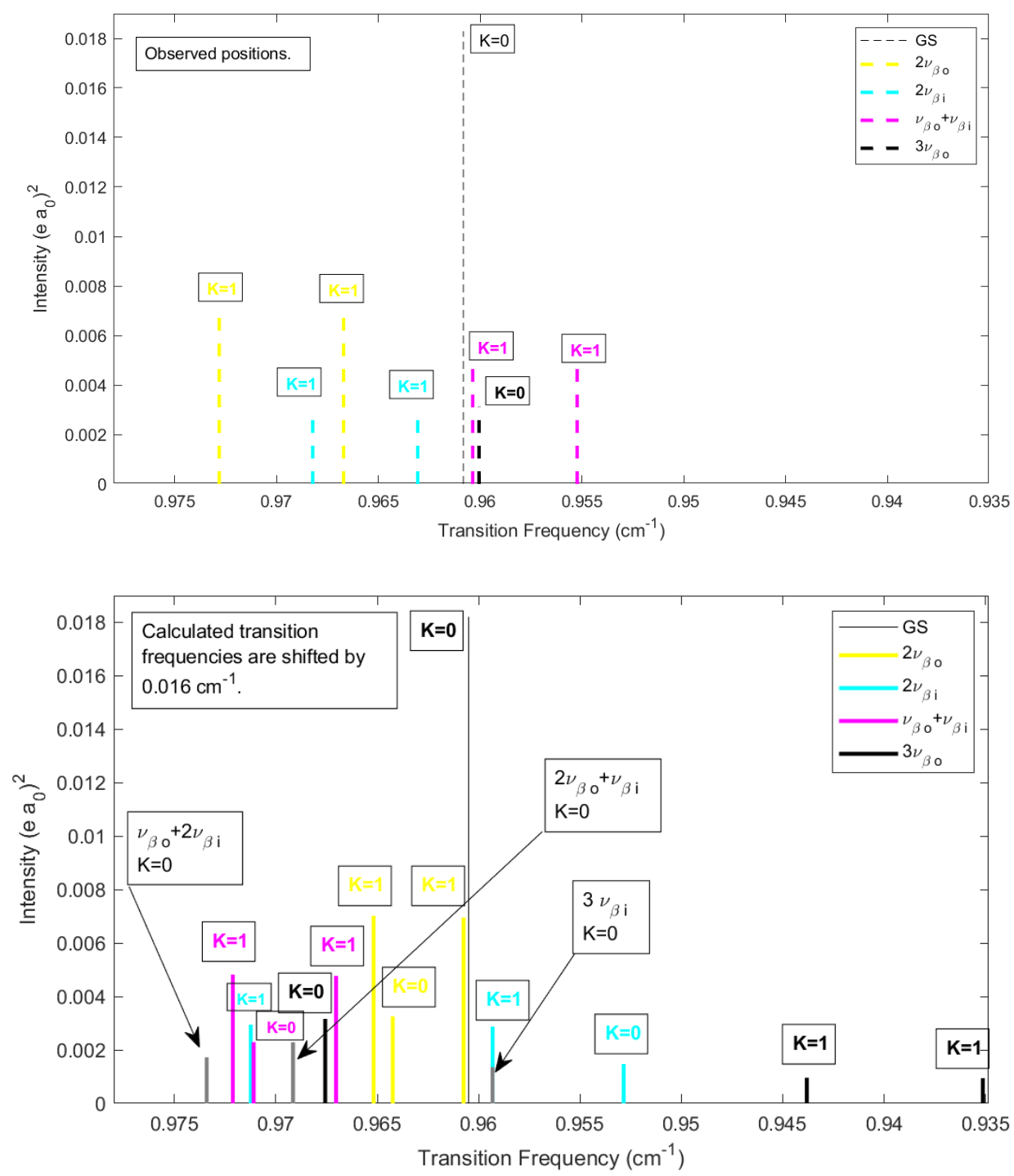

FIG. 11: Observed and calculated $R(1)$ transition frequencies in the $2 \nu_{\beta o}, 2 \nu_{\beta i}, \nu_{\beta o}+\nu_{\beta i}, 3 \nu_{\beta o}, \nu_{\beta o}+$ $2 \nu_{\beta i}, 2 \nu_{\beta o}+\nu_{\beta i}$ and $3 \nu_{\beta i}$ vibrational states. The stronger $R(1)$ lines of the ground state, $\nu_{\beta o}$, and $\nu_{\beta i}$ also fall into this region (shown in Fig. 10) but are removed for clarity except that we keep the $K=0 R(1)$ line of the ground state. Note that if Kisiel et al.'s assignment of satellite A(yellow) and $\mathrm{B}$ (purple) is interchanged, the order of the calculated yellow and purple lines would match that of the experiment. 

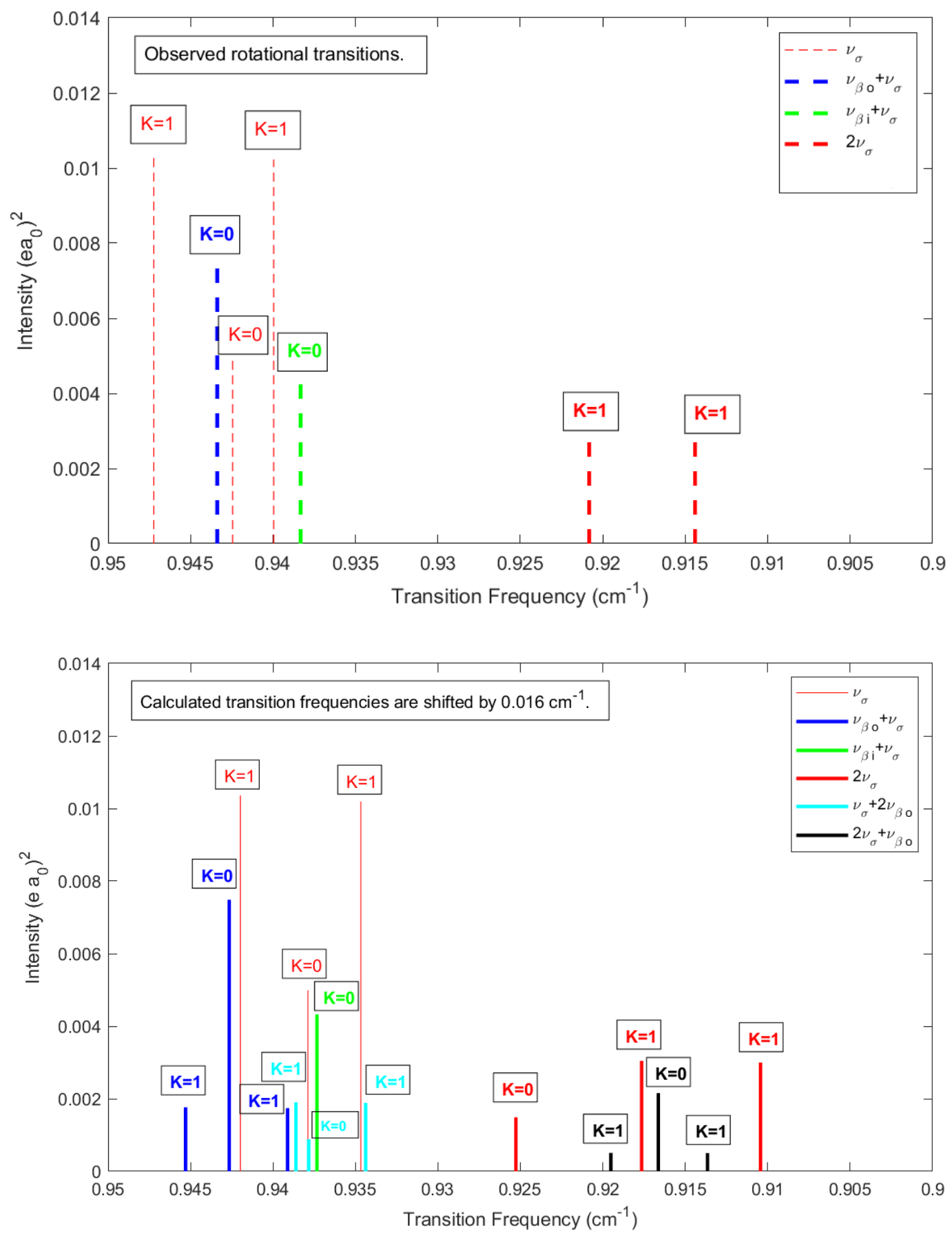

FIG. 12: Observed (top panel) and calculated (bottom panel) $J=2 \leftarrow 1$ rotational transitions in the $\nu_{\sigma}$ region. 
TABLE X: Comparison of vibrational levels with those of Ref. [20] (in $\mathrm{cm}^{-1}$ ). The Zero Point Energy is relative to the $5-\mathrm{D} D_{e}$.

\begin{tabular}{|c|c|c|c|c|c|}
\hline & K, Sym, State & $\mathrm{J}\left(K_{a}, K_{c}\right)$ & Ref.[20]'s calc. & Ref.[20]'s PES, our calc. & This work \\
\hline \multirow[t]{5}{*}{$\mathrm{pH}_{2} \mathrm{O}-\mathrm{HF}$} & $\mathrm{K}=0, A^{+}, \mathrm{GS}$ & $0(0,0)$ & $0.0(-2079.6)$ & $0.0(-2079.56)$ & $0.0(-1992.38)$ \\
\hline & $\mathrm{K}=2, A^{+}, \mathrm{GS}$ & $2(2,0)$ & 55.35 & 55.317 & 55.586 \\
\hline & $\mathrm{K}=2, A^{-}, \mathrm{GS}$ & $2(2,1)$ & 55.35 & 55.317 & 55.586 \\
\hline & $\mathrm{K}=1, A^{+}, \nu_{\beta}(o)$ & $1(1,1)$ & 90.07 & 90.054 & 92.921 \\
\hline & $\mathrm{K}=1, A^{-}, \nu_{\beta}(o)$ & $1(1,0)$ & 90.07 & 90.057 & 92.924 \\
\hline \multirow[t]{3}{*}{$\mathrm{oH}_{2} \mathrm{O}-\mathrm{HF}$} & $\mathrm{K}=1, B^{-}, \mathrm{GS}$ & $1(1,1)$ & 14.00 & 13.991 & 14.056 \\
\hline & $\mathrm{K}=1, B^{+}, \mathrm{GS}$ & $1(1,0)$ & 14.00 & 13.995 & 14.060 \\
\hline & $\mathrm{K}=0, B^{-}, \nu_{\beta}(o)$ & $0(0,0)$ & 78.92 & 78.920 & 81.862 \\
\hline
\end{tabular}

